\title{
Bilinear multipliers of weighted Lebesgue spaces and variable exponent Lebesgue spaces
}

\section{Öznur Kulak* and A Turan Gürkanlı}

\section{"Correspondence:}

oznurn@omu.edu.tr

Department of Mathematics,

Faculty of Arts and Sciences,

Ondokuz Mayıs University,

Kurupelit, Samsun, 55139, Turkey

\begin{abstract}
Let $1 \leq p_{1}, p_{2}<\infty, 0<p_{3} \leq \infty$ and $\omega_{1}, \omega_{2}, \omega_{3}$ be weight functions on $\mathbb{R}^{n}$. Assume that $\omega_{1}, \omega_{2}$ are slowly increasing functions.

We say that a bounded function $m(\xi, \eta)$ defined on $\mathbb{R}^{n} \times \mathbb{R}^{n}$ is a bilinear multiplier on $\mathbb{R}^{n}$ of type $\left(p_{1}, \omega_{1} ; p_{2}, \omega_{2} ; p_{3}, \omega_{3}\right)$ (shortly $\left.\left(\omega_{1}, \omega_{2}, \omega_{3}\right)\right)$ if

$$
B_{m}(f, g)(x)=\int_{\mathbb{R}^{n}} \int_{\mathbb{R}^{n}} \hat{f}(\xi) \hat{g}(\eta) m(\xi, \eta) e^{2 \pi i\langle\xi+\eta, x\rangle} d \xi d \eta
$$

is a bounded bilinear operator from $L_{\omega_{1}}^{p_{1}}\left(\mathbb{R}^{n}\right) \times L_{\omega_{2}}^{p_{2}}\left(\mathbb{R}^{n}\right)$ to $L_{\omega_{3}}^{p_{3}}\left(\mathbb{R}^{n}\right)$. We denote by $\operatorname{BM}\left(p_{1}, \omega_{1} ; p_{2}, \omega_{2} ; p_{3}, \omega_{3}\right)$ (shortly $\left.\operatorname{BM}\left(\omega_{1}, \omega_{2}, \omega_{3}\right)\right)$ the vector space of bilinear multipliers of type $\left(\omega_{1}, \omega_{2}, \omega_{3}\right)$.

In this paper first we discuss some properties of the space $B M\left(\omega_{1}, \omega_{2}, \omega_{3}\right)$. Furthermore, we give some examples of bilinear multipliers.

At the end of this paper, by using variable exponent Lebesgue spaces $L^{p_{1}(x)}\left(\mathbb{R}^{n}\right)$, $L^{p_{2}(x)}\left(\mathbb{R}^{n}\right)$ and $L^{p_{3}(x)}\left(\mathbb{R}^{n}\right)$, we define the space of bilinear multipliers from $L^{p_{1}(x)}\left(\mathbb{R}^{n}\right) \times L^{p_{2}(x)}\left(\mathbb{R}^{n}\right)$ to $L^{p_{3}(x)}\left(\mathbb{R}^{n}\right)$ and discuss some properties of this space.
\end{abstract}

MSC: 42A45; 42B15; 42B35

Keywords: bilinear multipliers; weighted Lebesgue space; variable exponent Lebesgue space

\section{Introduction}

Throughout this paper $C_{c}^{\infty}\left(\mathbb{R}^{n}\right), C_{c}\left(\mathbb{R}^{n}\right)$ and $S\left(\mathbb{R}^{n}\right)$ denote the space of infinitely differentiable complex-valued functions with compact support on $\mathbb{R}^{n}$, the space of all continuous, complex-valued functions with compact support on $\mathbb{R}^{n}$ and the space of infinitely differentiable complex-valued functions on $\mathbb{R}^{n}$ rapidly decreasing at infinity, respectively. For $1 \leq p \leq \infty, L^{p}\left(\mathbb{R}^{n}\right)$ denotes the usual Lebesgue space. A continuous function $\omega$ satisfying $1 \leq \omega(x)$ and $\omega(x+y) \leq \omega(x) \omega(y)$ for $x, y \in \mathbb{R}^{n}$ will be called a weight function on $\mathbb{R}^{n}$. If $\omega_{1}(x) \leq \omega_{2}(x)$ for all $x \in \mathbb{R}^{n}$, we say that $\omega_{1} \leq \omega_{2}$. We say that a weight function $v_{s}$ is of polynomial type if $v_{s}(x)=(1+|x|)^{s}$ for $s \geq 0$. Let $f$ be a measurable function on $\mathbb{R}^{n}$. If there exist $C>0$ and $N \in \mathbb{N}$ such that

$$
|f(x)| \leq C\left(1+x^{2}\right)^{N}
$$

\section{Springer}

○ 2013 Kulak and Gürkanli; licensee Springer. This is an Open Access article distributed under the terms of the Creative Commons Attribution License (http://creativecommons.org/licenses/by/2.0), which permits unrestricted use, distribution, and reproduction in any medium, provided the original work is properly cited. 
for all $x \in \mathbb{R}^{n}$, then $f$ is said to be a slowly increasing function. It is easy to see that polynomial-type weight functions are slowly increasing.

For $1 \leq p \leq \infty$, we set

$$
L_{\omega}^{p}\left(\mathbb{R}^{n}\right)=\left\{f: f \omega \in L^{p}\left(\mathbb{R}^{n}\right)\right\}
$$

It is known that $L_{\omega}^{p}\left(\mathbb{R}^{n}\right)$ is a Banach space under the norm

$$
\|f\|_{p, \omega}=\|f \omega\|_{p}=\left\{\int_{\mathbb{R}^{n}}|f(x) \omega(x)|^{p} d x\right\}^{\frac{1}{p}}, \quad 1 \leq p<\infty
$$

or

$$
\|f\|_{\infty, \omega}=\|f \omega\|_{\infty}=\underset{x \in \mathbb{R}^{n}}{\operatorname{ess} \sup }|f(x) \omega(x)|, \quad p=\infty,[1,2]
$$

The dual of the space $L_{\omega}^{p}\left(\mathbb{R}^{n}\right)$ is the space $L_{\omega^{-1}}^{q}\left(\mathbb{R}^{n}\right)$, where $\frac{1}{p}+\frac{1}{q}=1$ and $\omega^{-1}(x)=\frac{1}{\omega(x)}$. For $f \in L^{1}\left(\mathbb{R}^{n}\right)$, the Fourier transform of $f$ is denoted by $\hat{f}$. We know that $\hat{f}$ is a continuous function on $\mathbb{R}^{n}$, which vanishes at infinity, and it has the inequality $\|\hat{f}\|_{\infty} \leq\|f\|_{1}[3,4]$. Let $f$ be a measurable function on $\mathbb{R}^{n}$. The translation, character and dilation operators $T_{x}, M_{x}$ and $D_{s}$ are defined by $T_{x} f(y)=f(y-x), M_{x} f(y)=e^{2 \pi i\langle x, y\rangle} f(y)$ and $D_{t}^{p} f(y)=t^{-\frac{n}{p}} f\left(\frac{y}{t}\right)$ respectively for $x, y \in \mathbb{R}^{n}, 0<p, t<\infty$. With this notation out of the way one has, for $1 \leq$ $p \leq \infty$ and $\frac{1}{p}+\frac{1}{p^{\prime}}=1$,

$$
\left(T_{x} f\right)^{\wedge}(\xi)=M_{-x} \hat{f}(\xi), \quad\left(M_{x} f\right)^{\wedge}(\xi)=T_{x} \hat{f}(\xi), \quad\left(D_{t}^{p} f\right)^{\wedge}(\xi)=D_{t^{-1}}^{p^{\prime}} \hat{f}(\xi)
$$

We denote by $M\left(\mathbb{R}^{n}\right)$ the space of bounded regular Borel measures, by $M(\omega)$ the space of $\mu$ in $M\left(\mathbb{R}^{n}\right)$ such that

$$
\|\mu\|_{\omega}=\int_{\mathbb{R}^{n}} \omega d|\mu|<\infty .
$$

If $\mu \in M\left(\mathbb{R}^{n}\right)$, the Fourier-Stieltjes transform of $\mu$ is denoted by $\hat{\mu}$ [5]. In this paper, $P\left(\mathbb{R}^{n}\right)$ denotes the family of all measurable functions $p: \mathbb{R}^{n} \rightarrow[1, \infty)$. We put

$$
p_{*}=\underset{x \in \mathbb{R}^{n}}{\operatorname{essinf}} p(x), \quad p^{*}=\underset{x \in \mathbb{R}^{n}}{\operatorname{ess} \sup } p(x) .
$$

We shall also use the notation

$$
\Omega_{\infty}=\left\{x \in \mathbb{R}^{n}: p(x)=\infty\right\}
$$

The generalized Lebesgue space (or the variable exponent Lebesgue space) $L^{p(x)}\left(\mathbb{R}^{n}\right)$ is defined to be a space of (equivalence classes) measurable functions $f$ such that

$$
\varrho_{p}(\lambda f)=\int_{\mathbb{R}^{n} \backslash \Omega_{\infty}}|\lambda f(x)|^{p(x)} d x+\underset{x \in \Omega_{\infty}}{\operatorname{ess} \sup }(\lambda f(x))<\infty
$$


for some $\lambda=\lambda(f)>0$. If $p^{*}<\infty$, then

$$
\varrho_{p}(\lambda f)=\int_{\mathbb{R}^{n} \backslash \Omega_{\infty}}|\lambda f(x)|^{p(x)} d x, \quad[6,7]
$$

It is known by Theorem 2.5 in [6] that $L^{p(x)}\left(\mathbb{R}^{n}\right)$ is a Banach space with the Luxemburg norm

$$
\|f\|_{p(x)}=\inf \left\{\lambda>0: \varrho_{p}\left(\frac{f}{\lambda}\right) \leq 1\right\}
$$

If $p^{*}<\infty$, then $C_{c}^{\infty}\left(\mathbb{R}^{n}\right)$ is dense in $L^{p(x)}\left(\mathbb{R}^{n}\right)$. Also, if $p(x)=p$ is a constant function, then the above norm $\|\cdot\|_{p(x)}$ coincides with the usual norm $\|\cdot\|_{p}$. The vector space of locally integrable functions on $\mathbb{R}^{n}$ is denoted by $L_{\text {loc }}^{1}\left(\mathbb{R}^{n}\right)$. The space $L^{p(x)}\left(\mathbb{R}^{n}\right)$ is a solid space, that is, if $f \in L^{p(x)}\left(\mathbb{R}^{n}\right)$ is given and $g \in L_{\text {loc }}^{1}\left(\mathbb{R}^{n}\right)$ satisfies $|g(x)| \leq|f(x)|$ a.e., then $g \in L^{p(x)}\left(\mathbb{R}^{n}\right)$ and $\|g\|_{p(x)} \leq\|f\|_{p(x)}$ by [8]. In this paper we assume that $p^{*}<\infty$.

\section{The bilinear multipliers space $\operatorname{BM}\left(p_{1}, \omega_{1} ; p_{2}, \omega_{2} ; p_{3}, \omega_{3}\right)$}

Lemma 2.1 Let $1 \leq p<\infty$ and let $\omega$ be a slowly increasing weight function. Then $S\left(\mathbb{R}^{n}\right)$ is dense in $L_{\omega}^{p}\left(\mathbb{R}^{n}\right)$.

Proof Let $f \in S\left(\mathbb{R}^{n}\right)$ be given. Since $\omega$ is a slowly increasing weight function, there exist $C>0$ and $N \in \mathbb{N}$ such that

$$
|\omega(x)| \leq C\left(1+x^{2}\right)^{N}=m(x)
$$

for all $x \in \mathbb{R}^{n}$. Also, since $m$ is a polynomial, then by Proposition 19.2.2 in [9], we have $S\left(\mathbb{R}^{n}\right) \subset L_{m}^{p}\left(\mathbb{R}^{n}\right)$. Hence, by (2.1), we obtain $S\left(\mathbb{R}^{n}\right) \subset L_{m}^{p}\left(\mathbb{R}^{n}\right) \subset L_{\omega}^{p}\left(\mathbb{R}^{n}\right)$.

Now, we show that $C_{c}^{\infty}\left(\mathbb{R}^{n}\right)$ is dense $L_{m}^{p}\left(\mathbb{R}^{n}\right)$. Let $f \in L_{w}^{p}\left(\mathbb{R}^{n}\right)$ be given. Then $f m \in L^{p}\left(\mathbb{R}^{n}\right)$. Since $C_{c}^{\infty}\left(\mathbb{R}^{n}\right)$ is dense $L^{p}\left(\mathbb{R}^{n}\right)$ by [6], for given $\varepsilon>0$, there exists $g \in C_{c}^{\infty}\left(\mathbb{R}^{n}\right)$ such that

$$
\|f m-g\|_{p}<\varepsilon .
$$

Therefore, by using the inequality (2.2), we write

$$
\|f m-g\|_{p}=\left\|f-g m^{-1}\right\|_{p, m}<\varepsilon
$$

Also, since $m \neq 0$ and $m$ is a polynomial, we have $g m^{-1} \in C_{c}^{\infty}\left(\mathbb{R}^{n}\right)$. Thus, we have $\overline{C_{c}^{\infty}\left(\mathbb{R}^{n}\right)}=$ $L_{m}^{p}\left(\mathbb{R}^{n}\right)$. By using the inclusion $C_{c}^{\infty}\left(\mathbb{R}^{n}\right) \subset S\left(\mathbb{R}^{n}\right) \subset L_{m}^{p}\left(\mathbb{R}^{n}\right)$, we obtain $\overline{S\left(\mathbb{R}^{n}\right)}=L_{m}^{p}\left(\mathbb{R}^{n}\right)$.

Now, take any $f \in L_{\omega}^{p}\left(\mathbb{R}^{n}\right)$. Since $\overline{C_{c}\left(\mathbb{R}^{n}\right)}=\overline{L_{m}^{p}\left(\mathbb{R}^{n}\right)}=L_{\omega}^{p}\left(\mathbb{R}^{n}\right)$, there exists $g \in L_{m}^{p}\left(\mathbb{R}^{n}\right)$ such that

$$
\|f-g\|_{p, \omega}<\frac{\varepsilon}{2}
$$

Furthermore, since $S\left(\mathbb{R}^{n}\right)$ is dense $L_{m}^{p}\left(\mathbb{R}^{n}\right)$, there exists $h \in S\left(\mathbb{R}^{n}\right)$ such that

$$
\|g-h\|_{p, m}<\frac{\varepsilon}{2}
$$


Combining the inequalities (2.3) and (2.4), we have

$$
\|f-g\|_{p, \omega} \leq\|f-g\|_{p, \omega}+\|h-g\|_{p, \omega} \leq\|f-g\|_{p, \omega}+\|h-g\|_{p, m}<\varepsilon,
$$

which means $\overline{S\left(\mathbb{R}^{n}\right)}=L_{\omega}^{p}\left(\mathbb{R}^{n}\right)$.

Definition 2.1 Let $1 \leq p_{1}, p_{2}<\infty, 0<p_{3} \leq \infty$ and $\omega_{1}, \omega_{2}, \omega_{3}$ be weight functions on $\mathbb{R}^{n}$. Assume that $\omega_{1}, \omega_{2}$ are slowly increasing functions and $m(\xi, \eta)$ is a bounded function on $\mathbb{R}^{n} \times \mathbb{R}^{n}$. Define

$$
B_{m}(f, g)(x)=\int_{\mathbb{R}^{n}} \int_{\mathbb{R}^{n}} \hat{f}(\xi) \hat{g}(\eta) m(\xi, \eta) e^{2 \pi i\langle\xi+\eta, x\rangle} d \xi d \eta
$$

for all $f, g \in S\left(\mathbb{R}^{n}\right)$.

$m$ is said to be a bilinear multiplier on $\mathbb{R}^{n}$ of type $\left(p_{1}, \omega_{1} ; p_{2}, \omega_{2} ; p_{3}, \omega_{3}\right)$ (shortly $\left(\omega_{1}, \omega_{2}\right.$, $\left.\left.\omega_{3}\right)\right)$ if there exists $C>0$ such that

$$
\left\|B_{m}(f, g)\right\|_{p_{3}, \omega_{3}} \leq C\|f\|_{p_{1}, \omega_{1}}\|g\|_{p_{2}, \omega_{2}}
$$

for all $f, g \in S\left(\mathbb{R}^{n}\right)$. That means $B_{m}$ extends to a bounded bilinear operator from $L_{\omega_{1}}^{p_{1}}\left(\mathbb{R}^{n}\right) \times$ $L_{\omega_{2}}^{p_{2}}\left(\mathbb{R}^{n}\right)$ to $L_{\omega_{3}}^{p_{3}}\left(\mathbb{R}^{n}\right)$.

We denote by $\operatorname{BM}\left(p_{1}, \omega_{1} ; p_{2}, \omega_{2} ; p_{3}, \omega_{3}\right)$ (shortly $\left.\operatorname{BM}\left(\omega_{1}, \omega_{2}, \omega_{3}\right)\right)$ the space of all bilinear multipliers of type $\left(\omega_{1}, \omega_{2}, \omega_{3}\right)$ and $\|m\|_{\left(\omega_{1}, \omega_{2}, \omega_{3}\right)}=\left\|B_{m}\right\|$.

Theorem 2.1 Let $\frac{1}{p_{1}}+\frac{1}{p_{2}}=\frac{1}{p_{3}}$ and $\omega_{3} \leq \omega_{1}$. If $K \in L_{\omega_{3}}^{1}\left(\mathbb{R}^{n}\right)$, then $m(\xi, \eta)=\hat{K}(\xi-\eta)$ defines a bilinear multiplier and $\|m\|_{\left(\omega_{1}, \omega_{2}, \omega_{3}\right)} \leq\|K\|_{1, \omega_{3}}$.

Proof For $f, g \in S\left(\mathbb{R}^{n}\right)$, we have $f(x-y)=\int_{\mathbb{R}^{n}} \hat{f}(\xi) e^{2 \pi i\langle x-y, \xi\rangle} d \xi$ and $g(x+y)=\int_{\mathbb{R}^{n}} \hat{g}(\eta) \times$ $e^{2 \pi i(x+y, \eta\rangle} d \eta$. Thus, by the Fubini theorem, we write

$$
\begin{aligned}
B_{m}(f, g)(x) & =\int_{\mathbb{R}^{n}} \int_{\mathbb{R}^{n}} \hat{f}(\xi) \hat{g}(\eta) \hat{K}(\xi-\eta) e^{2 \pi i\langle\xi+\eta, x\rangle} d \xi d \eta \\
& =\int_{\mathbb{R}^{n}} \int_{\mathbb{R}^{n}} \hat{f}(\xi) \hat{g}(\eta)\left(\int_{\mathbb{R}^{n}} K(y) e^{-2 \pi i\langle\xi-\eta, y\rangle} d y\right) e^{2 \pi i\langle\xi+\eta, x\rangle} d \xi d \eta \\
& =\int_{\mathbb{R}^{n}} \int_{\mathbb{R}^{n}} \int_{\mathbb{R}^{n}} \hat{f}(\xi) \hat{g}(\eta) K(y) e^{-2 \pi i\langle\xi-\eta, y\rangle} e^{2 \pi i\langle\xi+\eta, x\rangle} d \xi d \eta d y .
\end{aligned}
$$

Since $f, g \in S\left(\mathbb{R}^{n}\right)$, we have $\hat{f}, \hat{g} \in S\left(\mathbb{R}^{n}\right) \subset L^{1}\left(\mathbb{R}^{n}\right)$. Hence, by (2.5), we obtain

$$
\begin{aligned}
B_{m}(f, g)(x) & =\int_{\mathbb{R}^{n}}\left(\int_{\mathbb{R}^{n}} \hat{f}(\xi) e^{2 \pi i(x-y, \xi)}\right)\left(\int_{\mathbb{R}^{n}} \hat{g}(\eta) e^{2 \pi i(x+y, \eta)} d \eta\right) K(y) d y \\
& =\int_{\mathbb{R}^{n}} f(x-y) g(x+y) K(y) d y .
\end{aligned}
$$

Since $\omega_{3} \leq \omega_{1}$, then

$$
\left\|f(x-y) \omega_{3}\right\|_{p_{1}} \leq \omega_{3}(y)\|f\|_{p_{1}, \omega_{1}}
$$


and hence $f(x-y) \omega_{3} \in L^{p_{1}}\left(\mathbb{R}^{n}\right)$. Therefore from (2.6) and the Minkowski inequality, we write

$$
\begin{aligned}
\left\|B_{m}(f, g)\right\|_{p_{3}, \omega_{3}} & \leq \int_{\mathbb{R}^{n}} \int_{\mathbb{R}^{n}}\|f(x-y) g(x+y)\|_{p_{3}, \omega_{3}}|K(y)| d y \\
& =\int_{\mathbb{R}^{n}} \int_{\mathbb{R}^{n}}\left\|f(x-y) g(x+y) \omega_{3}\right\|_{p_{3}}|K(y)| d y .
\end{aligned}
$$

Hence, using the generalized Hölder inequality and combining (2.7), (2.8), we have

$$
\begin{aligned}
\left\|B_{m}(f, g)\right\|_{p_{3}, \omega_{3}} & \leq \int_{\mathbb{R}^{n}}\left\|f(x-y) \omega_{3}\right\|_{p_{1}}\|g(x+y)\|_{p_{2}} \omega_{3}(y)|K(y)| d y \\
& \leq \int_{\mathbb{R}^{n}}\|f\|_{p_{1}}\|g\|_{p_{2}} \omega_{3}(y)|K(y)| d y \\
& \leq \int_{\mathbb{R}^{n}}\|f\|_{p_{1}, \omega_{1}}\|g\|_{p_{2}, \omega_{2}} \omega_{3}(y)|K(y)| d y \\
& =\|f\|_{p_{1}, \omega_{1}}\|g\|_{p_{2}, \omega_{2}}\|K\|_{1, \omega_{3}} .
\end{aligned}
$$

If we set $C=\|K\|_{1, \omega_{3}}$, we obtain

$$
\left\|B_{m}(f, g)\right\|_{p_{3}, \omega_{3}} \leq C\|f\|_{p_{1}, \omega_{1}}\|g\|_{p_{2}, \omega_{2}} .
$$

Then $m \in \operatorname{BM}\left(\omega_{1}, \omega_{2}, \omega_{3}\right)$. Consequently, using (2.9), we have

$$
\begin{aligned}
\|m\|_{\left(\omega_{1}, \omega_{2}, \omega_{3}\right)} & =\sup \left\{\frac{\left\|B_{m}(f, g)\right\|_{p_{3}, \omega_{3}}}{\|f\|_{p_{1}, \omega_{1}}\|g\|_{p_{2}, \omega_{2}}}:\|f\|_{p_{1}, \omega_{1}} \leq 1,\|g\|_{p_{2}, \omega_{2}} \leq 1\right\} \\
& \leq\|K\|_{1, \omega_{3}} .
\end{aligned}
$$

Definition 2.2 Let $1 \leq p_{1}, p_{2}<\infty, 0<p_{3} \leq \infty$ and $\omega_{1}, \omega_{2}, \omega_{3}$ be weight functions on $\mathbb{R}^{n}$. Suppose that $\omega_{1}, \omega_{2}$ are slowly increasing functions. We denote by $\tilde{M}\left(\omega_{1}, \omega_{2}, \omega_{3}\right)$ the space of measurable functions $M: \mathbb{R}^{n} \rightarrow \mathbb{C}$ such that $m(\xi, \eta)=M(\xi-\eta) \in \operatorname{BM}\left(\omega_{1}, \omega_{2}, \omega_{3}\right)$, that is to say,

$$
B_{M}(f, g)(x)=\int_{\mathbb{R}^{n}} \int_{\mathbb{R}^{n}} \hat{f}(\xi) \hat{g}(\eta) M(\xi-\eta) e^{2 \pi i\langle\xi+\eta, x\rangle} d \xi d \eta
$$

extends to a bounded bilinear map from $L_{\omega_{1}}^{p_{1}}\left(\mathbb{R}^{n}\right) \times L_{\omega_{2}}^{p_{2}}\left(\mathbb{R}^{n}\right)$ to $L_{\omega_{3}}^{p_{3}}\left(\mathbb{R}^{n}\right)$. We denote $\|M\|_{\left(\omega_{1}, \omega_{2}, \omega_{3}\right)}=\left\|B_{M}\right\|$.

Theorem 2.2 Let $p_{3} \geq 1$ and $\omega_{3}(-x)=\omega_{3}(x)$. Then $m \in \mathrm{BM}\left(\omega_{1}, \omega_{2}, \omega_{3}\right)$ if and only if there exists $C>0$ such that

$$
\left|\int_{\mathbb{R}^{n}} \int_{\mathbb{R}^{n}} \hat{f}(\xi) \hat{g}(\eta) \hat{h}(\xi+\eta) m(\xi, \eta) d \xi d \eta\right| \leq C\|f\|_{p_{1}, \omega_{1}}\|g\|_{p_{2}, \omega_{2}}\|h\|_{p_{3}^{\prime}, \omega_{3}^{-1}}
$$

for all $f, g, h \in S\left(\mathbb{R}^{n}\right)$, where $\frac{1}{p_{3}}+\frac{1}{p_{3}^{\prime}}=1$. 
Proof Let $m \in \operatorname{BM}\left(\omega_{1}, \omega_{2}, \omega_{3}\right)$. We take any $f, g, h \in S\left(\mathbb{R}^{n}\right)$. From the Fubini theorem, we write

$$
\begin{aligned}
& \left|\int_{\mathbb{R}^{n}} \int_{\mathbb{R}^{n}} \hat{f}(\xi) \hat{g}(\eta) \hat{h}(\xi+\eta) m(\xi, \eta) d \xi d \eta\right| \\
& \quad=\left|\int_{\mathbb{R}^{n}} \int_{\mathbb{R}^{n}} \hat{f}(\xi) \hat{g}(\eta)\left\{\int_{\mathbb{R}^{n}} h(y) e^{-2 \pi i\langle\xi+\eta, y\rangle} d y\right\} m(\xi, \eta) d \xi d \eta\right| \\
& \quad=\left|\int_{\mathbb{R}^{n}} h(y) B_{m}(f, g)(-y) d y\right|=\left|\int_{\mathbb{R}^{n}} h(y) \tilde{B}_{m}(f, g)(y) d y\right| \\
& \quad \leq \int_{\mathbb{R}^{n}}|h(y)|\left|\tilde{B}_{m}(f, g)(y)\right| d y,
\end{aligned}
$$

where $\tilde{B}_{m}(f, g)(y)=B_{m}(f, g)(-y)$. On the other hand, since $m \in \operatorname{BM}\left(\omega_{1}, \omega_{2}, \omega_{3}\right)$, then $B_{m}(f, g) \in L_{\omega_{3}}^{p_{3}}\left(\mathbb{R}^{n}\right)$. Thus we obtain $\tilde{B}_{m}(f, g) \in L_{\omega_{3}}^{p_{3}}\left(\mathbb{R}^{n}\right)$. Also, $h \in S\left(\mathbb{R}^{n}\right) \subset L^{p_{3}^{\prime}}\left(\mathbb{R}^{n}\right) \subset$ $L_{\omega_{3}^{-1}}^{p_{3}^{\prime}}\left(\mathbb{R}^{n}\right)$. Hence, using the Hölder inequality and the inequality (2.10), we write

$$
\begin{aligned}
& \left|\int_{\mathbb{R}^{n}} \int_{\mathbb{R}^{n}} \hat{f}(\xi) \hat{g}(\eta) \hat{h}(\xi+\eta) m(\xi, \eta) d \xi d \eta\right| \\
& \quad \leq \int_{\mathbb{R}^{n}}\left|h(y) \omega_{3}^{-1}(y) \| \tilde{B}_{m}(f, g)(y) \omega_{3}(y)\right| d y \\
& \quad \leq\left\|\tilde{B}_{m}(f, g)\right\|_{p_{3}, \omega_{3}}\|h\|_{p_{3}^{\prime}, \omega_{3}^{-1}}
\end{aligned}
$$

Moreover, since $m \in \operatorname{BM}\left(\omega_{1}, \omega_{2}, \omega_{3}\right)$, there exists $C>0$ such that

$$
\left\|B_{m}(f, g)\right\|_{p_{3}, \omega_{3}} \leq C\|f\|_{p_{1}, \omega_{1}}\|g\|_{p_{2}, \omega_{2}} .
$$

If we combine (2.11) and (2.12), we write

$$
\left|\int_{\mathbb{R}^{n}} \int_{\mathbb{R}^{n}} \hat{f}(\xi) \hat{g}(\eta) \hat{h}(\xi+\eta) m(\xi, \eta) d \xi d \eta\right| \leq C\|f\|_{p_{1}, \omega_{1}}\|g\|_{p_{2}, \omega_{2}}\|h\|_{p_{3}^{\prime}, \omega_{3}^{-1}}
$$

For the proof of converse, assume that there exists a constant $C>0$ such that

$$
\left|\int_{\mathbb{R}^{n}} \int_{\mathbb{R}^{n}} \hat{f}(\xi) \hat{g}(\eta) \hat{h}(\xi+\eta) m(\xi, \eta) d \xi d \eta\right| \leq C\|f\|_{p_{1}, \omega_{1}}\|g\|_{p_{2}, \omega_{2}}\|h\|_{p_{3}^{\prime}, \omega_{3}^{-1}}
$$

for all $f, g, h \in S\left(\mathbb{R}^{n}\right)$. From the assumption and (2.10), we write

$$
\left|\int_{\mathbb{R}^{n}} h(y) \tilde{B}_{m}(f, g)(y) d y\right| \leq C\|f\|_{p_{1}, \omega_{1}}\|g\|_{p_{2}, \omega_{2}}\|h\|_{p_{3}^{\prime}, \omega_{3}^{-1}} .
$$

Define a function $l$ from $S\left(\mathbb{R}^{n}\right) \subset L_{\omega_{3}^{-1}}^{p^{\prime}}\left(\mathbb{R}^{n}\right)$ to $\mathbb{C}$ such that

$$
\ell(h)=\int_{\mathbb{R}^{n}} h(y) \tilde{B}_{m}(f, g)(y) d y .
$$

It is clear that the function $\ell$ is linear and bounded by (2.13). By using $\overline{C_{c}\left(\mathbb{R}^{n}\right)}=L_{\omega_{3}^{-1}}^{p^{\prime}}\left(\mathbb{R}^{n}\right)$ in [10], it is easy to show that $\overline{C_{c}^{\infty}\left(\mathbb{R}^{n}\right)}=L_{\omega_{3}^{-1}}^{p_{3}^{\prime}}\left(\mathbb{R}^{n}\right)$. So, by the inclusion $C_{c}^{\infty}\left(\mathbb{R}^{n}\right) \subset S\left(\mathbb{R}^{n}\right) \subset$ 
$L_{\omega_{3}^{-1}}^{p^{\prime}}\left(\mathbb{R}^{n}\right)$, we have $\overline{S\left(\mathbb{R}^{n}\right)}=L_{\omega_{3}^{-1}}^{p^{\prime}}\left(\mathbb{R}^{n}\right)$. Thus $\ell$ extends to a bounded function from $L_{\omega_{3}^{-1}}^{p^{\prime}}\left(\mathbb{R}^{n}\right)$ to $\mathbb{C}$. Then $\ell \in\left(L_{\omega_{3}^{-1}}^{p_{3}^{\prime}}\left(\mathbb{R}^{n}\right)\right)^{*}=L_{\omega_{3}}^{p_{3}}\left(\mathbb{R}^{n}\right)$ and by $(2.13)$, we have

$$
\begin{aligned}
\left\|B_{m}(f, g)\right\|_{p_{3}, \omega_{3}} & =\left\|\tilde{B}_{m}(f, g)\right\|_{p_{3}, \omega_{3}}=\|\ell\|=\sup _{\|h\|_{p_{3}^{\prime}, \omega_{3}^{-1}} \leq 1} \frac{|l(h)|}{\|h\|_{p_{3}^{\prime}, \omega_{3}^{-1}}} \\
& \leq \sup _{\|h\|_{p_{3}^{\prime}, \omega_{3}^{-1}} \leq 1} \frac{C\|f\|_{p_{1}, \omega_{1}}\|g\|_{p_{2}, \omega_{2}}\|h\|_{p_{3}^{\prime}, \omega_{3}^{-1}}}{\|h\|_{p_{3}^{\prime}, \omega_{3}^{-1}}^{-1}} \leq C\|f\|_{p_{1}, \omega_{1}}\|g\|_{p_{2}, \omega_{2}} .
\end{aligned}
$$

Hence, we obtain $m \in \operatorname{BM}\left(\omega_{1}, \omega_{2}, \omega_{3}\right)$.

Theorem 2.3 Let $\frac{1}{p_{1}}+\frac{1}{p_{2}}=\frac{1}{p_{3}}, p_{3} \geq 1$ and $v_{s}(x)=(1+|x|)^{s}, s \geq 0$ be a weight function of polynomial type such that $v_{s} \leq \omega_{1}$. If $\mu \in M\left(v_{s}\right)$ and $m(\xi, \eta)=\hat{\mu}(\alpha \xi+\beta \eta)$ for $\alpha, \beta \in \mathbb{R}$, then $m \in \operatorname{BM}\left(\omega_{1}, \omega_{2}, v_{s}\right)$. Moreover,

$$
\begin{aligned}
& \|m\|_{\left(\omega_{1}, \omega_{2}, v_{s}\right)} \leq\|\mu\|_{v_{s}} \text { if }|\alpha| \leq 1, \\
& \|m\|_{\left(\omega_{1}, \omega_{2}, v_{s}\right)} \leq|\alpha|^{s}\|\mu\|_{v_{s}} \quad \text { if }|\alpha|>1 .
\end{aligned}
$$

Proof Let $f, g, \in S\left(\mathbb{R}^{n}\right)$. Then

$$
\begin{aligned}
B_{m}(f, g)(x) & =\int_{\mathbb{R}^{n}} \int_{\mathbb{R}^{n}} \hat{f}(\xi) \hat{g}(\eta) \hat{\mu}(\alpha \xi+\beta \eta) e^{2 \pi i\langle\xi+\eta, x\rangle} d \xi d \eta \\
& =\int_{\mathbb{R}^{n}} \int_{\mathbb{R}^{n}} \hat{f}(\xi) \hat{g}(\eta)\left\{\int_{\mathbb{R}^{n}} e^{-2 \pi i\langle\alpha \xi+\beta \eta, t\rangle} d \mu(t)\right\} e^{2 \pi i\langle\xi+\eta, x\rangle} d \xi d \eta \\
& =\int_{\mathbb{R}^{n}}\left\{\int_{\mathbb{R}^{n}} \hat{f}(\xi) e^{2 \pi i\langle x-\alpha t, \xi\rangle} d \xi\right\}\left\{\int_{\mathbb{R}^{n}} \hat{g}(\eta) e^{2 \pi i\langle x-\beta t, \eta\rangle} d \eta\right\} d \mu(t) \\
& =\int_{\mathbb{R}^{n}} f(x-\alpha t) g(x-\beta t) d \mu(t) .
\end{aligned}
$$

On the other hand, by the assumption $v_{s} \leq \omega_{1}$, it is easy to see that $f(x-\alpha t) v_{s} \in L^{p_{1}}\left(\mathbb{R}^{n}\right)$ and

$$
\left\|f(x-\alpha t) v_{s}\right\|_{p_{1}} \leq v_{s}(\alpha t)\|f\|_{p_{1}, \omega_{1}} .
$$

Also, $g(x-\beta t) \in L^{p_{2}}\left(\mathbb{R}^{n}\right)$. Then, by (2.14), (2.15) and the generalized Hölder inequality, we have

$$
\begin{aligned}
\left\|B_{m}(f, g)\right\|_{p_{3}, v_{s}} & \leq \int_{\mathbb{R}^{n}}\|f(x-\alpha t) g(x-\beta t)\|_{p_{3}, v_{s}} d|\mu|(t) \\
& \leq \int_{\mathbb{R}^{n}}\left\|f(x-\alpha t) v_{s}\right\|_{p_{1}}\|g(x-\beta t)\|_{p_{2}} d|\mu|(t) \\
& \leq \int_{\mathbb{R}^{n}} v_{s}(\alpha t)\|f\|_{p_{1}, \omega_{1}}\|g\|_{p_{2}} d|\mu|(t) \\
& \leq\|f\|_{p_{1}, \omega_{1}}\|g\|_{p_{2}, \omega_{2}} \int_{\mathbb{R}^{n}} v_{s}(\alpha t) d|\mu|(t) .
\end{aligned}
$$


Now, suppose that $|\alpha| \leq 1$. Then we write

$$
\begin{aligned}
\int_{\mathbb{R}^{n}} v_{s}(\alpha t) d|\mu|(t) & =\int_{\mathbb{R}^{n}}(1+|\alpha t|)^{s} d|\mu|(t) \\
& \leq \int_{\mathbb{R}^{n}}(1+|t|)^{s} d|\mu|(t)=\|\mu\|_{v_{s}}
\end{aligned}
$$

Hence by (2.16)

$$
\left\|B_{m}(f, g)\right\|_{p_{3}, v_{s}} \leq\|f\|_{p_{1}, \omega_{1}}\|g\|_{p_{2}, \omega_{2}}\|\mu\|_{v_{s}} .
$$

Thus $m \in \operatorname{BM}\left(\omega_{1}, \omega_{2}, v_{s}\right)$ and by (2.17), we have

$$
\|m\|_{\left(\omega_{1}, \omega_{2}, v_{s}\right)}=\sup \left\{\frac{\left\|B_{m}(f, g)\right\|_{p_{3}, v_{s}}}{\|f\|_{p_{1}, \omega_{1}}\|g\|_{p_{2}, \omega_{2}}}:\|f\|_{p_{1}, \omega_{1}} \leq 1,\|g\|_{p_{2}, \omega_{2}} \leq 1\right\} \leq\|\mu\|_{v_{s}} .
$$

Similarly, if $|\alpha|>1$, then we write

$$
\begin{aligned}
\int_{\mathbb{R}^{n}} v_{s}(\alpha t) d|\mu|(t) & <\int_{\mathbb{R}^{n}}(|\alpha|+|\alpha||t|)^{s} d|\mu|(t) \\
& =|\alpha|^{s} \int_{\mathbb{R}^{n}} v_{s}(t) d|\mu|(t)=|\alpha|^{s}\|\mu\|_{v_{s}} .
\end{aligned}
$$

Again, by (2.16) we have

$$
\left\|B_{m}(f, g)\right\|_{p_{3}, v_{s}} \leq|\alpha|^{s}\|f\|_{p_{1}, \omega_{1}}\|g\|_{p_{2}, \omega_{2}}\|\mu\|_{v_{s}}
$$

Hence, we obtain $m \in \operatorname{BM}\left(\omega_{1}, \omega_{2}, v_{s}\right)$ and by (2.18)

$$
\|m\|_{\left(\omega_{1}, \omega_{2}, v_{s}\right)}=\sup \left\{\frac{\left\|B_{m}(f, g)\right\|_{p_{3}, v_{s}}}{\|f\|_{p_{1}, \omega_{1}}\|g\|_{p_{2}, \omega_{2}}}:\|f\|_{p_{1}, \omega_{1}} \leq 1,\|g\|_{p_{2}, \omega_{2}} \leq 1\right\} \leq|\alpha|^{s}\|\mu\|_{v_{s}} .
$$

Theorem 2.4 Let $m \in \operatorname{BM}\left(\omega_{1}, \omega_{2}, \omega_{3}\right)$.

(a) $T_{\left(\xi_{0}, \eta_{0}\right)} m \in \operatorname{BM}\left(\omega_{1}, \omega_{2}, \omega_{3}\right)$ for each $\left(\xi_{0}, \eta_{0}\right) \in \mathbb{R}^{2 n}$ and

$$
\left\|T_{\left(\xi_{0}, \eta_{0}\right)} m\right\|_{\left(\omega_{1}, \omega_{2}, \omega_{3}\right)}=\|m\|_{\left(\omega_{1}, \omega_{2}, \omega_{3}\right)} .
$$

(b) $M_{\left(\xi_{0}, \eta_{0}\right)} m \in \operatorname{BM}\left(\omega_{1}, \omega_{2}, \omega_{3}\right)$ for each $\left(\xi_{0}, \eta_{0}\right) \in \mathbb{R}^{2 n}$ and

$$
\left\|M_{\left(\xi_{0}, \eta_{0}\right)} m\right\|_{\left(\omega_{1}, \omega_{2}, \omega_{3}\right)} \leq \omega_{1}\left(-\xi_{0}\right) \omega_{2}\left(-\eta_{0}\right)\|m\|_{\left(\omega_{1}, \omega_{2}, \omega_{3}\right)} .
$$

Proof (a) Let us take any $f \in L_{\omega_{1}}^{p_{1}}\left(\mathbb{R}^{n}\right)$ and $g \in L_{\omega_{2}}^{p_{2}}\left(\mathbb{R}^{n}\right)$. If we say that $\xi-\xi_{0}=u$ and $\eta-\eta_{0}=v$, then

$$
\begin{aligned}
B_{T_{\left(\xi_{0}, \eta_{0}\right)} m}(f, g)(x) & =\int_{\mathbb{R}^{n}} \int_{\mathbb{R}^{n}} \hat{f}(\xi) \hat{g}(\eta) T_{\left(\xi_{0}, \eta_{0}\right)} m(\xi, \eta) e^{2 \pi i\langle\xi+\eta, x\rangle} d \xi d \eta \\
& =\int_{\mathbb{R}^{n}} \int_{\mathbb{R}^{n}} \hat{f}\left(u+\xi_{0}\right) \hat{g}\left(v+\eta_{0}\right) m(u, v) e^{2 \pi i\left\langle u+\xi_{0}, x\right\rangle} e^{2 \pi i\left\langle v+\eta_{0}, x\right\rangle} d u d v \\
& =\int_{\mathbb{R}^{n}} \int_{\mathbb{R}^{n}} T_{-\xi_{0}} \hat{f}(u) T_{-\eta_{0}} \hat{g}(v) m(u, v) e^{2 \pi i\left\langle\xi_{0}+\eta_{0}, x\right\rangle} e^{2 \pi i\langle u+v, x\rangle} d u d v
\end{aligned}
$$


By (2.19), we have

$$
\begin{aligned}
B_{T_{\left(\xi_{0}, \eta_{0}\right)} m}(f, g)(x) & =\int_{\mathbb{R}^{n}} \int_{\mathbb{R}^{n}} T_{-\xi_{0}} \hat{f}(u) T_{-\eta_{0}} \hat{g}(v) e^{2 \pi i\left\langle\xi_{0}+\eta_{0}, x\right\rangle} e^{2 \pi i\langle u+v, x\rangle} m(u, v) d u d v \\
& =e^{2 \pi i\left\langle\xi_{0}+\eta_{0}, x\right\rangle} \int_{\mathbb{R}^{n}} \int_{\mathbb{R}^{n}}\left(M_{-\xi_{0}} f\right)^{\wedge}(u)\left(M_{-\eta_{0}} g\right)^{\wedge}(v) m(u, v) e^{2 \pi i\langle u+v, x\rangle} d u d v \\
& =e^{2 \pi i\left\langle\xi_{0}+\eta_{0}, x\right\rangle} B_{m}\left(M_{-\xi_{0}} f, M_{-\eta_{0}} g\right)(x) .
\end{aligned}
$$

Since $m \in \operatorname{BM}\left(\omega_{1}, \omega_{2}, \omega_{3}\right),\left\|M_{-\xi_{0}} f\right\|_{p_{1}, \omega_{1}}=\|f\|_{p_{1}, \omega_{1}}$ and $\left\|M_{-\eta_{0}} g\right\|_{p_{2}, \omega_{2}}=\|g\|_{p_{2}, \omega_{2}}$ are satisfied for all $f \in L_{\omega_{1}}^{p_{1}}\left(\mathbb{R}^{n}\right)$ and $g \in L_{\omega_{2}}^{p_{2}}\left(\mathbb{R}^{n}\right)$. Hence, by (2.20), we have

$$
\begin{aligned}
\left\|B_{T_{\left(\xi_{0}, \eta_{0}\right)} m}(f, g)\right\|_{p_{3}, \omega_{3}} & =\left\|e^{2 \pi i\left\langle\xi_{0}+\eta_{0}, x\right\rangle} B_{m}\left(M_{-\xi_{0}} f, M_{-\eta_{0}} g\right)\right\|_{p_{3}, \omega_{3}} \\
& \leq C\|f\|_{p_{1}, \omega_{1}}\|g\|_{p_{2}, \omega_{2}}
\end{aligned}
$$

for some $C>0$. Thus $T_{\left(\xi_{0}, \eta_{0}\right) m} \in \mathrm{BM}\left(\omega_{1}, \omega_{2}, \omega_{3}\right)$. Also, we obtain

$$
\begin{aligned}
\| & T_{\left(\xi_{0}, \eta_{0}\right)} m \|_{\left(\omega_{1}, \omega_{2}, \omega_{3}\right)} \\
& =\left\|B_{T_{\left(\xi_{0}, \eta_{0}\right)} m}\right\| \\
& =\sup \left\{\frac{\left\|B_{T_{\left(\xi_{0}, \eta_{0}\right)} m}(f, g)\right\|_{p_{3}, \omega_{3}}}{\|f\|_{p_{1}, \omega_{1}}\|g\|_{p_{2}, \omega_{2}}}:\|f\|_{p_{1}, \omega_{1}} \leq 1,\|g\|_{p_{2}, \omega_{2}} \leq 1\right\} \\
& =\sup \left\{\frac{\left\|B_{m}\left(M_{-\xi_{0}} f, M_{-\eta_{0}} g\right)\right\|_{p_{3}, \omega_{3}}}{\left\|M_{-\xi_{0}} f\right\|_{p_{1}, \omega_{1}}\left\|M_{-\eta_{0}} g\right\|_{p_{2}, \omega_{2}}}:\left\|M_{-\xi_{0}} f\right\|_{p_{1}, \omega_{1}} \leq 1,\left\|M_{-\eta_{0}} g\right\|_{p_{2}, \omega_{2}} \leq 1\right\} \\
& =\left\|B_{m}\right\|=\|m\|_{\left(\omega_{1}, \omega_{2}, \omega_{3}\right)} .
\end{aligned}
$$

(b) Let us rewrite the value $B_{m}(f, g)$ as follows:

$$
\begin{aligned}
B_{M_{\left(\xi_{0}, \eta_{0}\right)}}(f, g)(x) & =\int_{\mathbb{R}^{n}} \int_{\mathbb{R}^{n}} \hat{f}(\xi) \hat{g}(\eta) M_{\left(\xi_{0}, \eta_{0}\right)} m(\xi, \eta) e^{2 \pi i\langle\xi+\eta, x\rangle} d \xi d \eta \\
& =\int_{\mathbb{R}^{n}} \int_{\mathbb{R}^{n}} \hat{f}(\xi) \hat{g}(\eta) e^{\left.2 \pi i\left(\xi_{0}, \eta_{0}\right),(\xi, \eta)\right\rangle} m(\xi, \eta) e^{2 \pi i\langle\xi+\eta, x\rangle} d \xi d \eta \\
& =\int_{\mathbb{R}^{n}} \int_{\mathbb{R}^{n}} M_{\xi_{0}} \hat{f}(\xi) M_{\eta_{0}} \hat{g}(\eta) m(\xi, \eta) e^{2 \pi i\langle\xi+\eta, x\rangle} d \xi d \eta \\
& =\int_{\mathbb{R}^{n}} \int_{\mathbb{R}^{n}}\left(T_{-\xi_{0}} f\right)^{\wedge}(\xi)\left(T_{-\eta_{0}} g\right)^{\wedge}(\eta) m(\xi, \eta) e^{2 \pi i\langle\xi+\eta, x\rangle} d \xi d \eta \\
& =B_{m}\left(T_{-\xi_{0}} f, T_{-\eta_{0}} g\right)(x) .
\end{aligned}
$$

Also, the inequalities $\left\|T_{-\xi_{0}} f\right\|_{p_{1}, \omega_{1}} \leq \omega_{1}\left(-\xi_{0}\right)\|f\|_{p_{1}, \omega_{1}}$ and $\left\|T_{-\eta_{0}} g\right\|_{p_{2}, \omega_{2}} \leq \omega_{2}\left(-\eta_{0}\right)\|g\|_{p_{2}, \omega_{2}}$ are satisfied for all $f \in L_{\omega_{1}}^{p_{1}}\left(\mathbb{R}^{n}\right), g \in L_{\omega_{2}}^{p_{2}}\left(\mathbb{R}^{n}\right)$. Hence, since $m \in \operatorname{BM}\left(\omega_{1}, \omega_{2}, \omega_{3}\right)$, by $(2.21)$ we have

$$
\begin{aligned}
\left\|B_{\left.M_{\left(\xi_{0}, \eta_{0}\right)}\right)}(f, g)\right\|_{p_{3}, \omega_{3}} & =\left\|B_{m}\left(T_{-\xi_{0}} f, T_{-\eta_{0}} g\right)\right\|_{p_{3}, \omega_{3}} \leq\left\|B_{m}\right\|\left\|T_{-\xi_{0}} f\right\|_{p_{1}, \omega_{1}}\left\|T_{-\eta_{0}} g\right\|_{p_{2}, \omega_{2}} \\
& \leq \omega_{1}\left(-\xi_{0}\right) \omega_{2}\left(-\eta_{0}\right)\left\|B_{m}\right\|\|f\|_{p_{1}, \omega_{1}}\|g\|_{p_{2}, \omega_{2}} .
\end{aligned}
$$


Kulak and Gürkanlı Journal of Inequalities and Applications 2013, 2013:259

Page 10 of 21

Then $M_{\left(\xi_{0}, \eta_{0}\right)} m \in \mathrm{BM}\left(\omega_{1}, \omega_{2}, \omega_{3}\right)$, and by $(2.22)$ we obtain

$$
\begin{aligned}
\left\|M_{\left(\xi_{0}, \eta_{0}\right)} m\right\|_{\left(\omega_{1}, \omega_{2}, \omega_{3}\right)} & =\sup \left\{\frac{\left\|B_{M_{\left(\xi_{0}, \eta_{0}\right)}}(f, g)\right\|_{p_{3}, \omega_{3}}}{\|f\|_{p_{1}, \omega_{1}}\|g\|_{p_{2}, \omega_{2}}}:\|f\|_{p_{1}, \omega_{1}} \leq 1,\|g\|_{p_{2}, \omega_{2}} \leq 1\right\} \\
& \leq \omega_{1}\left(-\xi_{0}\right) \omega_{2}\left(-\eta_{0}\right)\|m\|_{\left(\omega_{1}, \omega_{2}, \omega_{3}\right)} .
\end{aligned}
$$

Lemma 2.2 If $v_{s}$ is a polynomial-type weight function and $f \in L_{v_{s}}^{p}\left(\mathbb{R}^{n}\right)$, then $D_{t}^{p} f \in L_{v_{s}}^{p}\left(\mathbb{R}^{n}\right)$.

Moreover,

$$
\begin{aligned}
& \left\|D_{t}^{p} f\right\|_{p, v_{s}} \leq\|f\|_{p, v_{s}} \quad \text { if } t \leq 1, \\
& \left\|D_{t}^{p} f\right\|_{p, v_{s}}<t^{s}\|f\|_{p, v_{s}} \quad \text { if } t>1 .
\end{aligned}
$$

Proof Let $v_{s}$ be a polynomial-type weight function and $f \in L_{v_{s}}^{p}\left(\mathbb{R}^{n}\right)$. Assume that $t \leq 1$. If we get $\frac{x}{t}=u$,

$$
\begin{aligned}
\left\|D_{t}^{p} f\right\|_{p, v_{s}} & =\left\{\int_{\mathbb{R}^{n}}\left|D_{t}^{p} f(x)\right|^{p} v_{s}(x)^{p} d x\right\}^{\frac{1}{p}} \\
& =\left\{\int_{\mathbb{R}^{n}}\left|t^{-\frac{n}{p}} f\left(\frac{x}{t}\right)\right|^{p}(1+|x|)^{s p} d x\right\}^{\frac{1}{p}}=\left\{\int_{\mathbb{R}^{n}}|f(u)|^{p}(1+|u t|)^{s p} d u\right\}^{\frac{1}{p}} \\
& \leq\left\{\int_{\mathbb{R}^{n}}|f(u)|^{p}(1+|u|)^{s p} d u\right\}^{\frac{1}{p}} \\
& =\|f\|_{p, v_{s}}<\infty .
\end{aligned}
$$

Thus we have $D_{t}^{p} f \in L_{v_{s}}^{p}\left(\mathbb{R}^{n}\right)$ and $\left\|D_{t}^{p} f\right\|_{p, v_{s}} \leq\|f\|_{p, v_{s}}$.

Now, assume that $t>1$. Similarly by $(2.23)$

$$
\begin{aligned}
\left\|D_{t}^{p} f\right\|_{p, v_{s}} & =\left\{\int_{\mathbb{R}^{n}}|f(u)|^{p}(1+|u t|)^{s p} d u\right\}^{\frac{1}{p}} \\
& <\left\{\int_{\mathbb{R}^{n}}|f(u)|^{p}(t+|u t|)^{s p} d u\right\}^{\frac{1}{p}}=t^{s}\left\{\int_{\mathbb{R}^{n}}|f(u)|^{p}(1+|u|)^{s p} d u\right\}^{\frac{1}{p}} \\
& =t^{s} \mid f \|_{p, v_{s}}<\infty .
\end{aligned}
$$

Hence $D_{t}^{p} f \in L_{v_{s}}^{p}\left(\mathbb{R}^{n}\right)$, and we also have $\left\|D_{t}^{p} f\right\|_{p, v_{s}}<t^{s}\|f\|_{p, v_{s}}$.

Theorem 2.5 Let $v_{s_{1}}, v_{s_{2}}, v_{s_{3}}$ be weight functions of polynomial type and let $m \in$ $\operatorname{BM}\left(v_{s_{1}}, v_{s_{2}}, v_{s_{3}}\right)$. If $\frac{2}{q}=\frac{1}{p_{1}}+\frac{1}{p_{2}}-\frac{1}{p_{3}}$ and $0<t<\infty$, then $D_{t}^{q} m \in \operatorname{BM}\left(v_{s_{1}}, v_{s_{2}}, v_{s_{3}}\right)$. Moreover, then

$$
\begin{aligned}
& \left\|D_{t}^{q} m\right\|_{\left(v_{s_{1}}, v_{s_{2}}, v_{s_{3}}\right)} \leq\left(\frac{1}{t}\right)^{s_{3}}\|m\|_{\left(v_{s_{1}}, v_{s_{2}}, v_{s_{3}}\right)} \quad \text { if } t \leq 1, \\
& \left\|D_{t}^{q} m\right\|_{\left(v_{s_{1}}, v_{s_{2}}, v_{s_{3}}\right)}<t^{s_{1}+s_{2}}\|m\|_{\left(v_{s_{1}}, v_{s_{2}}, v_{s_{3}}\right)} \quad \text { if } t>1 .
\end{aligned}
$$


Proof Let $f \in L_{v_{s_{1}}}^{p_{1}}\left(\mathbb{R}^{n}\right)$ and $g \in L_{v_{s_{2}}}^{p_{2}}\left(\mathbb{R}^{n}\right)$ be given. We know by Lemma 2.2 that $D_{t}^{p_{1}} f \in$ $L_{v_{s_{1}}}^{p_{1}}\left(\mathbb{R}^{n}\right)$ and $D_{t}^{p_{2}} g \in L_{v_{s_{2}}}^{p_{2}}\left(\mathbb{R}^{n}\right)$. If we get $\frac{\xi}{t}=u$ and $\frac{\eta}{t}=v$, we obtain

$$
\begin{aligned}
B_{D_{t}^{q} m}(f, g)(x) & =\int_{\mathbb{R}^{n}} \int_{\mathbb{R}^{n}} \hat{f}(\xi) \hat{g}(\eta) D_{t}^{q} m(\xi, \eta) e^{2 \pi i\langle\xi+\eta, x\rangle} d \xi d \eta \\
& =\int_{\mathbb{R}^{n}} \int_{\mathbb{R}^{n}} \hat{f}(t u) \hat{g}(t v) t^{-\frac{2 n}{q}} m(u, v) e^{2 \pi i\langle u+v, t x\rangle} t^{2 n} d u d v
\end{aligned}
$$

Hence, from the equality $\frac{2}{q}=\frac{1}{p_{1}}+\frac{1}{p_{2}}-\frac{1}{p_{3}}$, we have

$$
\begin{aligned}
B_{D_{t}^{q} m}(f, g)(x) & =\int_{\mathbb{R}^{n}} \int_{\mathbb{R}^{n}} \hat{f}(t u) \hat{g}(t v) t^{-n\left(\frac{1}{p_{1}}+\frac{1}{p_{2}}-\frac{1}{p_{3}}\right)} m(u, v) e^{2 \pi i(u+v, t x\rangle} t^{2 n} d u d v \\
& =\int_{\mathbb{R}^{n}} \int_{\mathbb{R}^{n}} t^{-n\left(1-\frac{1}{p_{1}^{\prime}}\right)} \hat{f}(t u) t^{-n\left(1-\frac{1}{p_{1}^{\prime}}\right)} \hat{g}(t v) t^{\frac{n}{p_{3}}} m(u, v) e^{2 \pi i\langle u+v, t x)} t^{2 n} d u d v \\
& =t^{\frac{n}{p_{3}}} \int_{\mathbb{R}^{n}} \int_{\mathbb{R}^{n}} D_{t^{-1}}^{p_{1}^{\prime}} \hat{f}(u) D_{t^{-1}}^{p_{2}^{\prime}} \hat{g}(v) m(u, v) e^{2 \pi i\langle u+v, t x\rangle} d u d v \\
& =t^{\frac{n}{p_{3}}} \int_{\mathbb{R}^{n}} \int_{\mathbb{R}^{n}}\left(D_{t}^{p_{1}} f\right)^{\wedge}(u)\left(D_{t}^{p_{2}} g\right)^{\wedge}(v) m(u, v) e^{2 \pi i\langle u+v, t x\rangle} d u d v \\
& =D_{t^{-1}}^{p_{3}} B_{m}\left(D_{t}^{p_{1}} f, D_{t}^{p_{2}} g\right)(x) .
\end{aligned}
$$

Assume that $t \leq 1$. Since $m \in B_{m}\left(v_{s_{1}}, v_{s_{2}}, v_{s_{3}}\right)$, by Lemma 2.2 and using equality (2.24), we obtain

$$
\begin{aligned}
\left\|B_{D_{t}^{q} m}(f, g)\right\|_{p_{3}, v_{s_{3}}} & =\left\|D_{t^{-1}}^{p_{3}} B_{m}\left(D_{t}^{p_{1}} f, D_{t}^{p_{2}} g\right)(x)\right\|_{p_{3}, v_{s_{3}}} \\
& \leq\left(\frac{1}{t}\right)^{s_{3}}\left\|B_{m}\left(D_{t}^{p_{1}} f, D_{t}^{p_{2}} g\right)(x)\right\|_{p_{3}, v_{s_{3}}} \\
& \leq\left(\frac{1}{t}\right)^{s_{3}}\left\|B_{m}\right\|\left\|D_{t}^{p_{1}} f\right\|_{p, v_{s_{1}}}\left\|D_{t}^{p_{2}} g\right\|_{p, v_{s_{2}}} \\
& \left.\leq\left(\frac{1}{t}\right)^{s_{3}}\|m\|_{\left(v_{s_{1}}, v_{s_{2}}, v_{s_{3}}\right.}\right)\|f\|_{p_{1}, v_{s_{1}}}\|g\|_{p_{2}, v_{s_{2}}} .
\end{aligned}
$$

Then $D_{t}^{q} m \in \mathrm{BM}\left(v_{s_{1}}, v_{s_{2}}, v_{s_{3}}\right)$, and by $(2.25)$

$$
\left\|D_{t}^{q} m\right\|_{\left(v_{s_{1}}, v_{s_{2}}, v_{s_{3}}\right)} \leq\left(\frac{1}{t}\right)^{s_{3}}\|m\|_{\left(v_{s_{1}}, v_{s_{2}}, v_{s_{3}}\right)} .
$$

Now let $t>1$. Again, since $m \in \operatorname{BM}\left(v_{s_{1}}, v_{s_{2}}, v_{s_{3}}\right)$, by Lemma 2.2 and using equality (2.24), we obtain

$$
\begin{aligned}
\left\|B_{D_{t}^{q} m}(f, g)\right\|_{p_{3}, v_{s_{3}}} & <\left\|B_{m}\left(D_{t}^{p_{1}} f, D_{t}^{p_{2}} g\right)\right\|_{p_{3}, v_{s_{3}}} \\
& \leq\left\|B_{m}\right\|\left\|D_{t}^{p_{1}} f\right\|_{p, v_{s_{1}}}\left\|D_{t}^{p_{2}} g\right\|_{p, v_{s_{2}}} \\
& <t^{s_{1}+s_{2}}\left\|B_{m}\right\|\|f\|_{p_{1}, v_{s_{1}}}\|g\|_{p_{2}, v_{s_{2}}} \\
& =t^{s_{1}+s_{2}}\|m\|_{\left(v_{s_{1}}, v_{s_{2}}, v_{s_{3}}\right)}\|f\|_{p_{1}, v_{s_{1}}}\|g\|_{p_{2}, v_{s_{2}}} .
\end{aligned}
$$


Thus $D_{t}^{q} m \in \mathrm{BM}\left(v_{s_{1}}, v_{s_{2}}, v_{s_{3}}\right)$ and by $(2.26)$

$$
\left\|D_{t}^{q} m\right\|_{\left(v_{s_{1}}, v_{s_{2}}, v_{s_{3}}\right)}<t^{s_{1}+s_{2}}\|m\|_{\left(v_{s_{1}}, v_{s_{2}}, v_{s_{3}}\right)} .
$$

Theorem 2.6 Let $v_{s_{1}}, v_{s_{2}}, v_{s_{3}}$ be weight functions of polynomial type and let $m \in$ $\operatorname{BM}\left(v_{s_{1}}, v_{s_{2}}, v_{s_{3}}\right)$ such that $m(t \xi, t \eta)=m(\xi, \eta)$ for any $t>0$, where $\frac{2}{q}=\frac{1}{p_{1}}+\frac{1}{p_{2}}-\frac{1}{p_{3}}$. Then

$$
\begin{aligned}
& \frac{2}{q}<\frac{s_{3}}{n} \quad \text { if } t<1, \\
& \frac{2}{q}>-\frac{s_{1}+s_{2}}{n} \quad \text { if } t>1 .
\end{aligned}
$$

Proof Take any $f \in L_{v_{s_{1}}}^{p_{1}}\left(\mathbb{R}^{n}\right), g \in L_{v_{s_{2}}}^{p_{2}}\left(\mathbb{R}^{n}\right)$. It is known by Theorem 2.5 that

$$
B_{D_{t}^{q} m}(f, g)(x)=D_{t^{-1}}^{p_{3}} B_{m}\left(D_{t}^{p_{1}} f, D_{t}^{p_{2}} g\right)(x), \quad x \in \mathbb{R}^{n} .
$$

On the other hand, using $m(t \xi, t \eta)=m(\xi, \eta)$ and changing the variables $t u=\xi, t v=\eta$, we note that

$$
\begin{aligned}
& D_{t^{-1}}^{p_{3}} B_{m}\left(D_{t}^{p_{1}} f, D_{t}^{p_{2}} g\right)(x) \\
& \quad=t^{\frac{n}{p_{3}}} \int_{\mathbb{R}^{n}}\left(D_{t}^{p_{1}} f\right)^{\wedge}(u)\left(D_{t}^{p_{2}} g\right)^{\wedge}(v) m(u, v) e^{2 \pi i\langle u+v, t x\rangle} d u d v \\
& =t^{\frac{n}{p_{3}}} \int_{\mathbb{R}^{n}} \int_{\mathbb{R}^{n}} D_{t^{-1}}^{p_{1}^{\prime}} \hat{f}(u) D_{t^{-1}}^{p_{2}^{\prime}} \hat{g}(v) m(u, v) e^{2 \pi i\langle u+v, t x\rangle} d u d v \\
& =t^{\frac{n}{p_{3}}} \int_{\mathbb{R}^{n}} \int_{\mathbb{R}^{n}} \hat{f}(\xi) \hat{g}(\eta) m\left(t^{-1} \xi, t^{-1} \eta\right) e^{2 \pi i\left\langle t^{-1} \xi+t^{-1} \eta, t x\right\rangle} t^{n\left(\frac{1}{p_{1}^{\prime}}+\frac{1}{p_{2}^{\prime}}\right)} t^{-2 n} d \xi d \eta \\
& =t^{\frac{n}{p_{3}}} \int_{\mathbb{R}^{n}} \int_{\mathbb{R}^{n}} \hat{f}(\xi) \hat{g}(\eta) m(\xi, \eta) e^{2 \pi i\langle u+v, x\rangle} t^{-n\left(\frac{1}{p_{1}}+\frac{1}{p_{2}}\right)} d \xi d \eta \\
& =t^{n\left(\frac{1}{p_{3}}-\frac{1}{p_{1}}-\frac{1}{p_{2}}\right)} B_{m}(f, g)(x) .
\end{aligned}
$$

Hence by (2.27) and (2.28), we have

$$
B_{m}(f, g)(x)=t^{-n\left(\frac{1}{p_{3}}-\frac{1}{p_{1}}-\frac{1}{p_{2}}\right)} B_{D_{t}^{q} m}(f, g)(x) .
$$

Since $D_{t}^{q} m=m$ for $t=1$, we let $t \neq 1$. Assume first that $t<1$. Also, since $m \in \operatorname{BM}\left(v_{s_{1}}, v_{s_{2}}\right.$, $\left.v_{s_{3}}\right)$, by Theorem 2.5 we have $D_{t}^{q} m \in \operatorname{BM}\left(v_{s_{1}}, v_{s_{2}}, v_{s_{3}}\right)$ and $\left\|D_{t}^{q} m\right\|_{\left(v_{s_{1}}, v_{s_{2}}, v_{s_{3}}\right)}<\left(\frac{1}{t}\right)^{s_{3}} \times$ $\|m\|_{\left(v_{s_{1}}, v_{s_{2}}, v_{s_{3}}\right)}$. Then by $(2.28)$

$$
\begin{aligned}
\left\|B_{m}(f, g)(x)\right\|_{p_{3}, v_{s_{3}}} & =t^{-n\left(\frac{1}{p_{3}}-\frac{1}{p_{1}}-\frac{1}{p_{2}}\right)}\left\|B_{D_{t}^{q}}(f, g)(x)\right\|_{p_{3}, v_{s_{3}}} \\
& \leq t^{-n\left(\frac{1}{p_{3}}-\frac{1}{p_{1}}-\frac{1}{p_{2}}\right)}\left\|D_{t}^{q} m\right\|_{\left(v_{s_{1}}, v_{s_{2}}, v_{s_{3}}\right)}\|f\|_{p_{1}, v_{s_{1}}}\|g\|_{p_{2}, v_{s_{2}}} \\
& <t^{-n\left(\frac{1}{p_{3}}-\frac{1}{p_{1}}-\frac{1}{p_{2}}\right)-s_{3}}\|m\|_{\left(v_{s_{1}}, v_{s_{2}}, v_{s_{3}}\right)}\|f\|_{p_{1}, v_{s_{1}}}\|g\|_{p_{2}, v_{s_{2}}} \\
& =t^{-n\left(\frac{1}{p_{3}}-\frac{1}{p_{1}}-\frac{1}{p_{2}}\right)-s_{3}}\left\|B_{m}\right\|\|f\|_{p_{1}, v_{s_{1}}}\|g\|_{p_{2}, v_{s_{2}}} .
\end{aligned}
$$


Thus,

$$
\left\|B_{m}\right\|<t^{-n\left(\frac{1}{p_{3}}-\frac{1}{p_{1}}-\frac{1}{p_{2}}\right)-s_{3}}\left\|B_{m}\right\|=t^{\frac{2 n}{q}-s_{3}}\left\|B_{m}\right\| .
$$

Hence $1<t^{\frac{2 n}{q}-s_{3}}$. Since $t<1$, we have $\frac{2 n}{q}-s_{3}<0$. Thus, we write $\frac{2}{q}<\frac{s_{3}}{n}$.

Assume now that $t>1$. Again, by Theorem 2.5, we have $D_{t}^{q} m \in \operatorname{BM}\left(v_{s_{1}}, v_{s_{2}}, v_{s_{3}}\right)$ and $\left\|D_{t}^{q} m\right\|_{\left(v_{s_{1}}, v_{s_{2}}, v_{s_{3}}\right)}<t^{s_{1}+s_{2}}\|m\|_{\left(v_{s_{1}}, v_{s_{2}}, v_{s_{3}}\right)}$. Similarly,

$$
\left\|B_{m}(f, g)(x)\right\|_{p_{3}, v_{s_{3}}}<t^{-n\left(\frac{1}{p_{3}}-\frac{1}{p_{1}}-\frac{1}{p_{2}}\right)+s_{1}+s_{2}}\left\|B_{m}\right\|\|f\|_{p_{1}, v_{s_{1}}}\|g\|_{p_{2}, v_{s_{2}}} .
$$

Thus, we have

$$
\left\|B_{m}\right\|<t^{-n\left(\frac{1}{p_{3}}-\frac{1}{p_{1}}-\frac{1}{p_{2}}\right)+s_{1}+s_{2}}\left\|B_{m}\right\|=t^{\frac{2 n}{q}+s_{1}+s_{2}}\left\|B_{m}\right\| .
$$

Hence $1<t^{\frac{2 n}{q}+s_{1}+s_{2}}$ Since $t>1$, we have $\frac{2 n}{q}+s_{1}+s_{2}>0$. Thus, we write $\frac{2}{q}>-\frac{s_{1}+s_{2}}{n}$.

Theorem 2.7 Let $m \in \mathrm{BM}\left(\omega_{1}, \omega_{2}, \omega_{3}\right)$ and $p_{3} \geq 1$.

(a) If $\Phi \in L^{1}\left(\mathbb{R}^{n}\right)$, then $\Phi * m \in \mathrm{BM}\left(\omega_{1}, \omega_{2}, \omega_{3}\right)$ and

$$
\|\Phi * m\|_{\left(\omega_{1}, \omega_{2}, \omega_{3}\right)} \leq\|\Phi\|_{1}\|m\|_{\left(\omega_{1}, \omega_{2}, \omega_{3}\right)} .
$$

(b) If $\Phi \in L_{\omega}^{1}\left(\mathbb{R}^{n}\right)$ such that $\omega(u, v)=\omega_{1}(u) \omega_{2}(v)$, then $\hat{\Phi} m \in \operatorname{BM}\left(\omega_{1}, \omega_{2}, \omega_{3}\right)$ and

$$
\|\hat{\Phi} m\|_{\left(\omega_{1}, \omega_{2}, \omega_{3}\right)} \leq\|\Phi\|_{1, \omega}\|m\|_{\left(\omega_{1}, \omega_{2}, \omega_{3}\right)} .
$$

Proof (a) Let $f \in L_{\omega_{1}}^{p_{1}}\left(\mathbb{R}^{n}\right)$ and $g \in L_{\omega_{2}}^{p_{2}}\left(\mathbb{R}^{n}\right)$. Since $L_{\omega_{1}}^{p_{1}}\left(\mathbb{R}^{n}\right) \subset L^{p_{1}}\left(\mathbb{R}^{n}\right)$ and $L_{\omega_{2}}^{p_{2}}\left(\mathbb{R}^{n}\right) \subset L^{p_{2}}\left(\mathbb{R}^{n}\right)$, then by Proposition 2.5 in [11]

$$
B_{\Phi * m}(f, g)(x)=\int_{\mathbb{R}^{n}} \int_{\mathbb{R}^{n}} \Phi(u, v) B_{T_{(u, v)}}(f, g)(x) d u d v .
$$

Also, since $m \in \mathrm{BM}\left(\omega_{1}, \omega_{2}, \omega_{3}\right)$, we have $T_{(u, v)} m \in \mathrm{BM}\left(\omega_{1}, \omega_{2}, \omega_{3}\right)$ by Theorem 2.4. So, we write

$$
\begin{aligned}
\left\|B_{\Phi * m}(f, g)\right\|_{p_{3}, \omega_{3}} & \leq \int_{\mathbb{R}^{n}} \int_{\mathbb{R}^{n}}\left\|\Phi(u, v) B_{T_{(u, v)} m}(f, g)\right\|_{p_{3}, \omega_{3}} d u d v \\
& \leq \int_{\mathbb{R}^{n}} \int_{\mathbb{R}^{n}}|\Phi(u, v)|\left\|T_{(u, v)} m\right\|_{\left(\omega_{1}, \omega_{2}, \omega_{3}\right)}\|f\|_{p_{1}, \omega_{1}}\|g\|_{p_{2}, \omega_{2}} d u d v \\
& =\|m\|_{\left(\omega_{1}, \omega_{2}, \omega_{3}\right)}\|\Phi\|_{1}\|f\|_{p_{1}, \omega_{1}}\|g\|_{p_{2}, \omega_{2}}<\infty .
\end{aligned}
$$

Hence $\Phi * m \in \mathrm{BM}\left(\omega_{1}, \omega_{2}, \omega_{3}\right)$. Finally, by (2.29), we obtain

$$
\|\Phi * m\|_{\left(\omega_{1}, \omega_{2}, \omega_{3}\right)} \leq\|\Phi\|_{1}\|m\|_{\left(\omega_{1}, \omega_{2}, \omega_{3}\right)} .
$$

(b) Let $\Phi \in L_{\omega}^{1}\left(\mathbb{R}^{n}\right)$. Take any $f \in L_{\omega_{1}}^{p_{1}}\left(\mathbb{R}^{n}\right)$ and $g \in L_{\omega_{2}}^{p_{2}}\left(\mathbb{R}^{n}\right)$. It is known by Proposition 2.5 in [11] that the equality

$$
B_{\hat{\Phi} m}(f, g)(x)=\int_{\mathbb{R}^{n}} \int_{\mathbb{R}^{n}} \Phi(u, v) B_{M_{(-u,-v)} m}(f, g)(x) d u d v .
$$


Since $m \in \operatorname{BM}\left(\omega_{1}, \omega_{2}, \omega_{3}\right)$, by Theorem 2.4 we have $M_{(-u,-v)} m \in \operatorname{BM}\left(\omega_{1}, \omega_{2}, \omega_{3}\right)$ and

$$
\left\|M_{(-u,-v)} m\right\|_{\left(\omega_{1}, \omega_{2}, \omega_{3}\right)} \leq \omega_{1}(u) \omega_{2}(v)\|m\|_{\left(\omega_{1}, \omega_{2}, \omega_{3}\right)} .
$$

Then we write

$$
\begin{aligned}
\left\|B_{\hat{\Phi} m}(f, g)\right\|_{p_{3}, \omega_{3}} & \leq \int_{\mathbb{R}^{n}} \int_{\mathbb{R}^{n}}\left\|\Phi(u, v) B_{M_{(-u,-v)}}(f, g)\right\|_{p_{3}, \omega_{3}} d u d v \\
& \leq \int_{\mathbb{R}^{n}} \int_{\mathbb{R}^{n}}|\Phi(u, v)|\left\|M_{(-u,-v)} m\right\|_{\left(\omega_{1}, \omega_{2}, \omega_{3}\right)}\|f\|_{p_{1}, \omega_{1}}\|g\|_{p_{2}, \omega_{2}} d u d v \\
& \leq \int_{\mathbb{R}^{n}} \int_{\mathbb{R}^{n}}|\Phi(u, v)| \omega_{1}(u) \omega_{2}(v)\|m\|_{\left(\omega_{1}, \omega_{2}, \omega_{3}\right)}\|f\|_{p_{1}, \omega_{1}}\|g\|_{p_{2}, \omega_{2}} d u d v \\
& =\|m\|_{\left(\omega_{1}, \omega_{2}, \omega_{3}\right)}\|f\|_{p_{1}, \omega_{1}}\|g\|_{p_{2}, \omega_{2}}\|\Phi\|_{1, \omega} .
\end{aligned}
$$

Thus from (2.30), we obtain $\hat{\Phi} m \in \mathrm{BM}\left(\omega_{1}, \omega_{2}, \omega_{3}\right)$ and

$$
\|\hat{\Phi} m\|_{\left(\omega_{1}, \omega_{2}, \omega_{3}\right)} \leq\|\Phi\|_{1, \omega}\|m\|_{\left(\omega_{1}, \omega_{2}, \omega_{3}\right)} .
$$

Theorem 2.8 Let $v_{s_{1}}, v_{s_{2}}, v_{s_{3}}$ be weight functions of polynomial type and let $m \in$ $\operatorname{BM}\left(v_{s_{1}}, v_{s_{2}}, v_{s_{3}}\right)$. If $\Psi \in L^{1}\left(\mathbb{R}^{+}, t^{-\frac{2 n}{q}} d t\right)$ such that $\frac{2}{q}=\frac{1}{p_{1}}+\frac{1}{p_{2}}-\frac{1}{p_{3}}$, then $m_{\Psi}(\xi, \eta)=$ $\int_{0}^{\infty} m(t \xi, t \eta) \Psi(t) d t \in \operatorname{BM}\left(v_{s_{1}}, v_{s_{2}}, v_{s_{3}}\right)$. Moreover,

$$
\left\|m_{\Psi}\right\|_{\left(v_{s_{1}}, v_{s_{2}}, v_{s_{3}}\right)}<\|\Psi\|_{L^{1}\left(\mathbb{R}^{+}, t^{-\frac{2 n}{q}} d t\right)}\|m\|_{\left(v_{s_{1}}, v_{s_{2}}, v_{s_{3}}\right)} .
$$

Proof Let us take $f, g \in S\left(\mathbb{R}^{n}\right)$. Then

$$
\begin{aligned}
B_{m_{\Psi}}(f, g)(x) & =\int_{\mathbb{R}^{n}} \int_{\mathbb{R}^{n}} \hat{f}(\xi) \hat{g}(\eta) m_{\Psi}(\xi, \eta) e^{2 \pi i\langle u+v, x\rangle} d \xi d \eta \\
& =\int_{\mathbb{R}^{n}} \int_{\mathbb{R}^{n}} \hat{f}(\xi) \hat{g}(\eta)\left\{\int_{0}^{\infty} m(t \xi, t \eta) \Psi(t) d t\right\} e^{2 \pi i\langle u+v, x\rangle} d \xi d \eta \\
& =\int_{\mathbb{R}^{n}} \int_{\mathbb{R}^{n}} \hat{f}(\xi) \hat{g}(\eta)\left\{\int_{0}^{\infty} D_{t^{-1}}^{q} m(\xi, \eta) \Psi(t) t^{-\frac{2 n}{q}} d t\right\} e^{2 \pi i\langle u+v, x\rangle} d \xi d \eta \\
& =\int_{0}^{\infty} B_{D_{t^{-1}} m}(f, g) \Psi(t) t^{-\frac{2 n}{q}} d t .
\end{aligned}
$$

Since $m \in \operatorname{BM}\left(v_{s_{1}}, v_{s_{2}}, v_{s_{3}}\right), D_{t^{-1}}^{q} m \in \mathrm{BM}\left(v_{s_{1}}, v_{s_{2}}, v_{s_{3}}\right)$ by Theorem 2.5 , thus we observe that

$$
\begin{aligned}
\left\|B_{m_{\Psi}}(f, g)(x)\right\|_{p_{3}, v_{s_{3}}} \leq & \int_{0}^{\infty}\left\|B_{D_{t^{-1}}^{q} m}(f, g)\right\|_{p_{3}, v_{s_{3}}}|\Psi(t)| t^{-\frac{2 n}{q}} d t \\
\leq & \int_{0}^{\infty}\left\|B_{D_{t^{-1}}^{q} m}\right\|\|f\|_{p_{1}, v_{s_{1}}}\|g\|_{p_{2}, v_{s_{2}}}|\Psi(t)| t^{-\frac{2 n}{q}} d t \\
= & \int_{0}^{\infty}\left\|D_{t^{-1}}^{q} m\right\|_{\left(v_{s_{1}}, v_{s_{2}}, v_{s_{3}}\right)}\|f\|_{p_{1}, v_{s_{1}}}\|g\|_{p_{2}, v_{s_{2}}}|\Psi(t)| t^{-\frac{2 n}{q}} d t \\
< & \int_{0}^{1} t^{s_{3}}\|m\|_{\left(v_{s_{1}}, v_{s_{2}}, v_{s_{3}}\right)}\|f\|_{p_{1}, v_{s_{1}}}\|g\|_{p_{2}, v_{s_{2}}}|\Psi(t)| t^{-\frac{2 n}{q}} d t \\
& +\int_{1}^{\infty} t^{-s_{1}-s_{2}}\|m\|_{\left(v_{s_{1}}, v_{s_{2}}, v_{s_{3}}\right)}\|f\|_{p_{1}, v_{s_{1}}}\|g\|_{p_{2}, v_{s_{2}}}|\Psi(t)| t^{-\frac{2 n}{q}} d t
\end{aligned}
$$




$$
\begin{aligned}
= & \|m\|_{\left(v_{s_{1}}, v_{s_{2}}, v_{s_{3}}\right)}\|f\|_{p_{1}, v_{s_{1}}}\|g\|_{p_{2}, v_{s_{2}}} \\
& \times\left\{\int_{0}^{1} t^{s_{3}}|\Psi(t)| t^{-\frac{2 n}{q}} d t+\int_{1}^{\infty} t^{-s_{1}-s_{2}}|\Psi(t)| t^{-\frac{2 n}{q}} d t\right\} .
\end{aligned}
$$

Also, since $t^{s_{3}} \leq 1$ for $s_{3} \geq 0, t \leq 1$ and $t^{-s_{1}-s_{2}}<1$ for $-s_{1}-s_{2} \leq 0, t>1$, by (2.31)

$$
\left\|B_{m_{\Psi}}(f, g)(x)\right\|_{p_{3}, v_{s_{3}}}<\|\Psi\|_{L^{1}\left(\mathbb{R}^{+}, t^{-\frac{2 n}{q}} d t\right)}\|m\|_{\left(v_{s_{1}}, v_{s_{2}}, v_{s_{3}}\right)}\|f\|_{p_{1}, v_{s_{1}}}\|g\|_{p_{2}, v_{s_{2}}} .
$$

Hence, $m_{\Psi} \in \operatorname{BM}\left(v_{s_{1}}, v_{s_{2}}, v_{s_{3}}\right)$ and

$$
\left\|m_{\Psi}\right\|_{\left(v_{s_{1}}, v_{s_{2}}, v_{s_{3}}\right)}<\|\Psi\|_{L^{1}\left(\mathbb{R}^{+}, t^{-\frac{2 n}{q}} d t\right)}\|m\|_{\left(v_{s_{1}}, v_{s_{2}}, v_{s_{3}}\right)} .
$$

Theorem 2.9 Let $p_{3} \geq 1$ and $m \in \operatorname{BM}\left(\omega_{1}, \omega_{2}, \omega_{3}\right)$. If $Q_{1}, Q_{2}$ are bounded measurable sets in $\mathbb{R}^{n}$, then

$$
h(\xi, \eta)=\frac{1}{\mu\left(Q_{1} \times Q_{2}\right)} \iint_{Q_{1} \times Q_{2}} m(\xi+u, \eta+v) d u d v \in \operatorname{BM}\left(\omega_{1}, \omega_{2}, \omega_{3}\right) .
$$

Proof Take any $f, g \in S\left(\mathbb{R}^{n}\right)$. Then we write

$$
\begin{aligned}
& B_{h}(f, g)(x) \\
& \quad=\int_{\mathbb{R}^{n}} \int_{\mathbb{R}^{n}} \hat{f}(\xi) \hat{g}(\eta) h(\xi, \eta) e^{2 \pi i\langle\xi+\eta, x\rangle} d \xi d \eta \\
& \quad=\frac{1}{\mu\left(Q_{1} \times Q_{2}\right)} \iint_{Q_{1} \times Q_{2}}\left\{\int_{\mathbb{R}^{n}} \int_{\mathbb{R}^{n}} \hat{f}(\xi) \hat{g}(\eta) m(\xi+u, \eta+v) e^{2 \pi i\langle\xi+\eta, x\rangle} d \xi d \eta\right\} d u d v \\
& =\frac{1}{\mu\left(Q_{1} \times Q_{2}\right)} \iint_{Q_{1} \times Q_{2}} B_{T_{(-u,-v) m}}(f, g)(x) d u d v .
\end{aligned}
$$

By using Theorem 2.4, we have

$$
\begin{aligned}
\left\|B_{h}(f, g)\right\|_{p_{3}, \omega_{3}} & \leq \frac{1}{\mu\left(Q_{1} \times Q_{2}\right)} \iint_{Q_{1} \times Q_{2}}\left\|B_{T_{(-u,-v) m}}(f, g)\right\|_{p_{3}, \omega_{3}} d u d v \\
& \leq \frac{1}{\mu\left(Q_{1} \times Q_{2}\right)} \iint_{Q_{1} \times Q_{2}}\left\|T_{(-u,-v) m}\right\|_{\left(\omega_{1}, \omega_{2}, \omega_{3}\right)}\|f\|_{p_{1}, \omega_{1}}\|g\|_{p_{2}, \omega_{2}} d u d v \\
& =\frac{1}{\mu\left(Q_{1} \times Q_{2}\right)}\|m\|_{\left(\omega_{1}, \omega_{2}, \omega_{3}\right)}\|f\|_{p_{1}, \omega_{1}}\|g\|_{p_{2}, \omega_{2}} \mu\left(Q_{1} \times Q_{2}\right) \\
& \left.=\|m\|_{\left(\omega_{1}, \omega_{2}, \omega_{3}\right)}\right) f f\left\|_{p_{1}, \omega_{1}}\right\| g \|_{p_{2}, \omega_{2}} .
\end{aligned}
$$

Hence, we obtain $h(\xi, \eta) \in \operatorname{BM}\left(\omega_{1}, \omega_{2}, \omega_{3}\right)$.

Theorem 2.10 Let $\omega(u, v)=\omega_{1}(u) \omega_{2}(v), \omega_{3} \leq \omega_{1}, \omega_{3}(-u)=\omega_{3}(u)$ and $\frac{1}{p_{1}}+\frac{1}{p_{2}}=\frac{1}{p_{3}} \leq 1$. Assume that $\Phi \in L_{\omega}^{1}\left(\mathbb{R}^{2 n}\right), \Psi_{1} \in L_{\omega_{1}}^{p_{1}}\left(\mathbb{R}^{n}\right)$ and $\Psi_{2} \in L_{\omega_{2}}^{p_{2}}\left(\mathbb{R}^{n}\right)$. If $m(\xi, \eta)=\hat{\Psi}_{1}(\xi) \hat{\Phi}(\xi, \eta) \hat{\Psi}_{2}(\eta)$, then $m \in \mathrm{BM}\left(1, \omega_{1} ; 1, \omega_{2} ; p_{3}, \omega_{3}\right)$. 
Proof For the proof we will use Theorem 2.2. Take any $f, g, h \in S\left(\mathbb{R}^{n}\right)$. Then

$$
\begin{aligned}
& \left|\int_{\mathbb{R}^{n}} \int_{\mathbb{R}^{n}} \hat{f}(\xi) \hat{g}(\eta) \hat{h}(\xi+\eta) m(\xi, \eta) d \xi d \eta\right| \\
& \quad=\left|\int_{\mathbb{R}^{n}} h(y)\left\{\int_{\mathbb{R}^{n}} \int_{\mathbb{R}^{n}} \hat{f}(\xi) \hat{g}(\eta) \hat{\Psi}_{1}(\xi) \hat{\Phi}(\xi, \eta) \hat{\Psi}_{2}(\eta) e^{-2 \pi i\langle\xi+\eta, x\rangle} d \xi d \eta\right\} d y\right| \\
& \quad \leq \int_{\mathbb{R}^{n}}\left|h(y) \omega_{3}^{-1}(y) B_{\hat{\Phi}}\left(f * \Psi_{1}, g * \Psi_{2}\right)(-y) \omega_{3}(y)\right| d y .
\end{aligned}
$$

Since the spaces $L_{\omega_{1}}^{p_{1}}\left(\mathbb{R}^{n}\right)$ and $L_{\omega_{2}}^{p_{2}}\left(\mathbb{R}^{n}\right)$ are Banach convolution module over the spaces $L_{\omega_{1}}^{1}\left(\mathbb{R}^{n}\right), L_{\omega_{2}}^{1}\left(\mathbb{R}^{n}\right)$ respectively, we write $f * \Psi_{1} \in L_{\omega_{1}}^{p_{1}}\left(\mathbb{R}^{n}\right)$ and $g * \Psi_{2} \in L_{\omega_{2}}^{p_{2}}\left(\mathbb{R}^{n}\right)$. Also, by Theorem 2.7, $\hat{\Phi} \in \operatorname{BM}\left(p_{1}, \omega_{1} ; p_{2}, \omega_{2} ; p_{3}, \omega_{3}\right)$. Therefore we obtain $B_{\hat{\Phi}}\left(f * \Psi_{1}, g * \Psi_{2}\right) \in L_{\omega_{3}}^{p_{3}}\left(\mathbb{R}^{n}\right)$. By using the Hölder inequality and the inequality (2.32), we find

$$
\begin{aligned}
& \left|\int_{\mathbb{R}^{n}} \int_{\mathbb{R}^{n}} \hat{f}(\xi) \hat{g}(\eta) \hat{h}(\xi+\eta) m(\xi, \eta) d \xi d \eta\right| \\
& \leq\|h\|_{p_{3}^{-1}, \omega_{3}^{-1}}\left\|B_{\hat{\Phi}}\left(f * \Psi_{1}, g * \Psi_{2}\right)\right\|_{p_{3}, \omega_{3}} \\
& \leq\|h\|_{p_{3}^{-1, \omega_{3}^{-1}}}\left\|B_{\hat{\Phi}}\right\|\|f\|_{1, \omega_{1}}\left\|\Psi_{1}\right\|_{p_{1}, \omega_{1}}\|g\|_{1, \omega_{2}}\left\|\Psi_{2}\right\|_{p_{2}, \omega_{2}} .
\end{aligned}
$$

If we say $C=\left\|B_{\hat{\Phi}}\right\|\left\|\Psi_{1}\right\|_{p_{1}, \omega_{1}}\left\|\Psi_{2}\right\|_{p_{2}, \omega_{2}}$, then we obtain

$$
\left|\int_{\mathbb{R}^{n}} \int_{\mathbb{R}^{n}} \hat{f}(\xi) \hat{g}(\eta) \hat{h}(\xi+\eta) m(\xi, \eta) d \xi d \eta\right| \leq C\|f\|_{1, \omega}\|g\|_{1, \omega_{2}}\|h\|_{p_{3}^{-1}, \omega_{3}^{-1}}
$$

which means $m \in \mathrm{BM}\left(1, \omega_{1} ; 1, \omega_{2} ; p_{3}, \omega_{3}\right)$.

The following theorem can be proved easily by using the technique of the proof in Theorem 2.10.

Theorem 2.11 Let $\omega(u, v)=\omega_{1}(u) \omega_{2}(v), \omega_{3} \leq \omega_{1}, \omega_{3}(-u)=\omega_{3}(u)$ and $\frac{1}{p_{1}}+\frac{1}{p_{2}}=\frac{1}{p_{3}} \leq 1$. If $m(\xi, \eta)=\hat{\Psi}_{1}(\xi) \hat{\Phi}(\xi, \eta) \hat{\Psi}_{2}(\eta)$ such that $\Phi \in L_{\omega}^{1}\left(\mathbb{R}^{2 n}\right), \Psi_{1} \in L_{\omega_{1}}^{1}\left(\mathbb{R}^{n}\right)$ and $\Psi_{2} \in L_{\omega_{2}}^{1}\left(\mathbb{R}^{n}\right)$, then $m \in \operatorname{BM}\left(p_{1}, \omega_{1} ; p_{2}, \omega_{2} ; p_{3}, \omega_{3}\right)$.

\section{The bilinear multipliers space $\mathrm{BM}\left(p_{1}(x), p_{2}(x), p_{3}(x)\right)$}

Definition 3.1 Let $p_{1}(x), p_{2}(x), p_{3}(x) \in P\left(\mathbb{R}^{n}\right)$ and let $p_{1}^{*}<\infty, p_{2}^{*}<\infty, p_{3}^{*}<\infty$. Assume that $m(\xi, \eta)$ is a bounded function on $\mathbb{R}^{n} \times \mathbb{R}^{n}$. Define

$$
B_{m}(f, g)(x)=\int_{\mathbb{R}^{n}} \int_{\mathbb{R}^{n}} \hat{f}(\xi) \hat{g}(\eta) m(\xi, \eta) e^{2 \pi i(\xi+\eta, x)} d \xi d \eta
$$

for all $f, g \in C_{c}^{\infty}\left(\mathbb{R}^{n}\right)$.

$m$ is said to be a bilinear multiplier on $\mathbb{R}^{n}$ of type $\left(p_{1}(x), p_{2}(x), p_{3}(x)\right)$ if there exists $C>0$ such that

$$
\left\|B_{m}(f, g)\right\|_{p_{3}(x)} \leq C\|f\|_{p_{1}(x)}\|g\|_{p_{2}(x)}
$$

for all $f, g \in C_{c}^{\infty}\left(\mathbb{R}^{n}\right)$, i.e., $B_{m}$ extends to a bounded bilinear operator from $L^{p_{1}(x)}\left(\mathbb{R}^{n}\right) \times$ $L^{p_{2}(x)}\left(\mathbb{R}^{n}\right)$ to $L^{p_{3}(x)}\left(\mathbb{R}^{n}\right)$. We denote by $\operatorname{BM}\left(p_{1}(x), p_{2}(x), p_{3}(x)\right)$ the space of bilinear multipliers of type $\left(p_{1}(x), p_{2}(x), p_{3}(x)\right)$ and $\|m\|_{\left(p_{1}(x), p_{2}(x), p_{3}(x)\right)}=\left\|B_{m}\right\|$. 
The following theorem can be proved easily by using the technique of the proof in Theorem 2.2.

Theorem 3.1 Let $p_{3}(-x)=p_{3}(x)$ and $\frac{1}{p_{3}(x)}+\frac{1}{q(x)}=1$ for all $x \in \mathbb{R}^{n}$. Then $m \in \operatorname{BM}\left(p_{1}(x)\right.$, $\left.p_{2}(x), p_{3}(x)\right)$ if and only if there exists $C>0$ such that

$$
\left|\int_{\mathbb{R}^{n}} \int_{\mathbb{R}^{n}} \hat{f}(\xi) \hat{g}(\eta) \hat{h}(\xi+\eta) m(\xi, \eta) d \xi d \eta\right| \leq C\|f\|_{p_{1}(x)}\|g\|_{p_{2}(x)}\|h\|_{q(x)}
$$

for all $f, g, h \in C_{c}^{\infty}\left(\mathbb{R}^{n}\right)$.

Theorem 3.2 Let $\frac{1}{p(x)}+\frac{1}{q(x)}=\frac{1}{r}$. If $\Phi \in L^{1}\left(\mathbb{R}^{n}\right)$, then $m(\xi, \eta)=\hat{\Phi}(\xi+\eta) \in \operatorname{BM}(p(x), q(x), r)$.

Proof Take any $f, g, h \in C_{c}^{\infty}\left(\mathbb{R}^{n}\right)$. Then

$$
\begin{aligned}
& \left|\int_{\mathbb{R}^{n}} \int_{\mathbb{R}^{n}} \hat{f}(\xi) \hat{g}(\eta) \hat{h}(\xi+\eta) \hat{\Phi}(\xi+\eta) d \xi d \eta\right| \\
& \quad=\left|\int_{\mathbb{R}^{n}} \int_{\mathbb{R}^{n}} \hat{f}(\xi) \hat{g}(\eta)(h * \Phi)^{\wedge}(\xi+\eta) d \xi d \eta\right| \\
& \quad=\left|\int_{\mathbb{R}^{n}}(h * \Phi)(x)\left\{\int_{\mathbb{R}^{n}} \int_{\mathbb{R}^{n}} \hat{f}(\xi) \hat{g}(\eta) e^{-2 \pi i\langle\xi+\eta, x\rangle} d \xi d \eta\right\} d x\right| \\
& \quad \leq \int_{\mathbb{R}^{n}}|(h * \Phi)(x)|\left|\tilde{B}_{1}(f, g)(x)\right| d x .
\end{aligned}
$$

Since the space $L^{r^{\prime}}\left(\mathbb{R}^{n}\right)$ is the Banach convolution module over $L^{1}\left(\mathbb{R}^{n}\right)$ such that $\frac{1}{r}+\frac{1}{r^{\prime}}=1$, we write $h * \Phi \in L^{r^{\prime}}\left(\mathbb{R}^{n}\right)$. Also, we have $1 \in \mathrm{BM}(p(x), q(x), r)$. Then by (3.1), we find $C_{1}>0$ such that

$$
\left|\int_{\mathbb{R}^{n}} \int_{\mathbb{R}^{n}} \hat{f}(\xi) \hat{g}(\eta) \hat{h}(\xi+\eta) \hat{\Phi}(\xi+\eta) d \xi d \eta\right| \leq C_{1}\|h\|_{r^{\prime}}\|\Phi\|_{1}\|f\|_{p(x)}\|g\|_{q(x)} .
$$

If we set $C=C_{1}\|\Phi\|_{1}$, we obtain

$$
\left|\int_{\mathbb{R}^{n}} \int_{\mathbb{R}^{n}} \hat{f}(\xi) \hat{g}(\eta) \hat{h}(\xi+\eta) \hat{\Phi}(\xi+\eta) d \xi d \eta\right| \leq C\|f\|_{p(x)}\|g\|_{q(x)}\|h\|_{r^{\prime}}
$$

and $m(\xi, \eta)=\hat{\Phi}(\xi+\eta) \in \operatorname{BM}(p(x), q(x), r)$.

Theorem 3.3 If $m \in \mathrm{BM}\left(p_{1}(x), p_{2}(x), p_{3}(x)\right)$, then $T_{\left(\xi_{0}, \eta_{0}\right)} m \in \mathrm{BM}\left(p_{1}(x), p_{2}(x), p_{3}(x)\right)$ and

$$
\left\|T_{\left(\xi_{0}, \eta_{0}\right)} m\right\|_{\left(p_{1}(x), p_{2}(x), p_{3}(x)\right)}=\|m\|_{\left(p_{1}(x), p_{2}(x), p_{3}(x)\right)}
$$

for all $\left(\xi_{0}, \eta_{0}\right) \in \mathbb{R}^{2 n}$

Proof Let us take any $f, g \in C_{c}^{\infty}\left(\mathbb{R}^{n}\right)$. By the proof of (a) Theorem 2.4, we know that

$$
B_{T_{\left(\xi_{0}, \eta_{0}\right)} m}(f, g)(x)=e^{2 \pi i\left\langle\xi_{0}+\eta_{0}, x\right\rangle} B_{m}\left(M_{-\xi_{0}} f, M_{-\eta_{0}} g\right)(x), \quad x \in \mathbb{R}^{n} .
$$


By Lemma 5 in [8], we know $\left\|M_{-\xi_{0}} f\right\|_{p_{1}(x)}=\|f\|_{p_{1}(x)}$ and $\left\|M_{-\eta_{0}} g\right\|_{p_{2}(x)}=\|g\|_{p_{2}(x)}$. Since $m \in$ $\operatorname{BM}\left(p_{1}(x), p_{2}(x), p_{3}(x)\right)$, by (3.2), there exists $C>0$ such that

$$
\left\|B_{T_{\left(\xi_{0}, \eta_{0}\right)} m}(f, g)\right\|_{p_{3}(x)}=\left\|B_{m}\left(M_{-\xi_{0}} f, M_{-\eta_{0}} g\right)\right\|_{p_{3}(x)} \leq C\|f\|_{p_{1}(x)}\|g\|_{p_{2}(x)} .
$$

Thus $T_{\left(\xi_{0}, \eta_{0}\right)} m \in \mathrm{BM}\left(p_{1}(x), p_{2}(x), p_{3}(x)\right)$. Moreover, by using the same technique as in the proof of Theorem 2.4, we obtain

$$
\left\|T_{\left(\xi_{0}, \eta_{0}\right)} m\right\|_{\left(p_{1}(x), p_{2}(x), p_{3}(x)\right)}=\|m\|_{\left(p_{1}(x), p_{2}(x), p_{3}(x)\right)} .
$$

Theorem 3.4 Let $\frac{1}{p(x)}+\frac{1}{q(x)}=\frac{1}{r(x)}$. If $m \in \mathrm{BM}(p(x), q(x), r(x))$, then $\Phi * m \in \operatorname{BM}(p(x), q(x)$, $r(x))$ and there exists $C>0$ such that

$$
\|\Phi * m\|_{(p(x), q(x), r(x))} \leq C\|\Phi\|_{1}\|m\|_{(p(x), q(x), r(x))}
$$

for all $\Phi \in L^{1}\left(\mathbb{R}^{2 n}\right)$.

Proof Take any $f, g \in C_{c}^{\infty}\left(\mathbb{R}^{n}\right)$. By Proposition 2.5 in [11], we know that

$$
B_{\Phi * m}(f, g)(x)=\int_{\mathbb{R}^{n}} \int_{\mathbb{R}^{n}} \Phi(u, v) B_{T_{\left(\xi_{0}, \eta_{0}\right)} m}(f, g)(x) d u d v .
$$

Since $m \in \operatorname{BM}(p(x), q(x), r(x))$, then $T_{\left(\xi_{0}, \eta_{0}\right)} m \in \operatorname{BM}(p(x), q(x), r(x))$ and

$$
\left\|T_{\left(\xi_{0}, \eta_{0}\right)} m\right\|_{(p(x), q(x), r(x))}=\|m\|_{(p(x), q(x), r(x))}
$$

by Theorem 3.3. Using (3.3) and the Minkowski inequality for a variable exponent Lebesgue space [12], we find $C>0$ such that

$$
\begin{aligned}
\left\|B_{\Phi * m}(f, g)\right\|_{r(x)} & \leq C \int_{\mathbb{R}^{n}} \int_{\mathbb{R}^{n}}|\Phi(u, v)|\left\|B_{T_{\left(\xi_{0}, \eta_{0}\right)} m}(f, g)\right\|_{r(x)} d u d v \\
& \leq C \int_{\mathbb{R}^{n}} \int_{\mathbb{R}^{n}}|\Phi(u, v)|\left\|B_{T_{\left(\xi_{0}, \eta_{0}\right)} m}\right\|\|f\|_{p(x)}\|g\|_{q(x)} d u d v \\
& =C \int_{\mathbb{R}^{n}} \int_{\mathbb{R}^{n}}|\Phi(u, v)|\|m\|_{(p(x), q(x), r(x))}\|f\|_{p(x)}\|g\|_{q(x)} d u d v \\
& =C\|m\|_{(p(x), q(x), r(x))}\|f\|_{p(x)}\|g\|_{q(x)}\|\Phi\|_{1} .
\end{aligned}
$$

Hence $\Phi * m \in \operatorname{BM}(p(x), q(x), r(x))$ and by (3.4), we have

$$
\|\Phi * m\|_{(p(x), q(x), r(x))} \leq C\|\Phi\|_{1}\|m\|_{(p(x), q(x), r(x))} .
$$

Theorem 3.5 Let $r(-x)=r(x)$.

(a) If $\Psi_{1} \in L^{p}\left(\mathbb{R}^{n}\right), \Psi_{2} \in L^{q}\left(\mathbb{R}^{n}\right)$ and $m \in \operatorname{BM}(p, q, r(x))$, then $\hat{\Psi}_{1}(\xi) m(\xi, \eta) \hat{\Psi}_{2}(\eta) \in \mathrm{BM}(1,1, r(x))$.

(b) If $\Psi_{1}, \Psi_{2} \in L^{1}\left(\mathbb{R}^{n}\right)$ and $m \in \operatorname{BM}(p, q, r(x))$, then $\hat{\Psi}_{1}(\xi) m(\xi, \eta) \hat{\Psi}_{2}(\eta) \in \operatorname{BM}(p, q, r(x))$.

(c) If $\Psi_{1} \in L^{p}\left(\mathbb{R}^{n}\right)$ and $m \in \mathrm{BM}(p, q, r(x))$, then $\hat{\Psi}_{1}(\xi) m(\xi, \eta) \in \mathrm{BM}(1, q(x), r(x))$.

(d) If $\Psi_{1} \in L^{1}\left(\mathbb{R}^{n}\right)$ and $m \in \operatorname{BM}(p, q, r(x))$, then $\hat{\Psi}_{1}(\xi) m(\xi, \eta) \in \operatorname{BM}(p, q(x), r(x))$. 
Proof (a) Let $f, g, h \in C_{c}^{\infty}\left(\mathbb{R}^{n}\right)$ be given. Then

$$
\begin{aligned}
& \left|\int_{\mathbb{R}^{n}} \int_{\mathbb{R}^{n}} \hat{f}(\xi) \hat{g}(\eta) \hat{h}(\xi+\eta) \hat{\Psi}_{1}(\xi) m(\xi, \eta) \hat{\Psi}_{2}(\eta) d \xi d \eta\right| \\
& \quad=\left|\int_{\mathbb{R}^{n}} h(y)\left\{\int_{\mathbb{R}^{n}} \int_{\mathbb{R}^{n}} \hat{f}(\xi) \hat{g}(\eta) \hat{\Psi}_{1}(\xi) m(\xi, \eta) \hat{\Psi}_{2}(\eta) e^{-2 \pi i\langle\xi+\eta, y\rangle} d \xi d \eta\right\} d y\right| \\
& \quad=\left|\int_{\mathbb{R}^{n}} h(y)\left\{\int_{\mathbb{R}^{n}} \int_{\mathbb{R}^{n}}\left(f * \Psi_{1}\right)^{\wedge}(\xi)\left(g * \Psi_{2}\right)^{\wedge}(\eta) m(\xi, \eta) e^{-2 \pi i\langle\xi+\eta, y\rangle} d \xi d \eta\right\} d y\right| \\
& \quad \leq \int_{\mathbb{R}^{n}}|h(y)|\left|\tilde{B}_{m}\left(f * \Psi_{1}, g * \Psi_{2}\right)(y)\right| d x .
\end{aligned}
$$

Since the spaces $L^{p}\left(\mathbb{R}^{n}\right)$ and $L^{q}\left(\mathbb{R}^{n}\right)$ are Banach convolution module over $L^{1}\left(\mathbb{R}^{n}\right)$, we have $f * \Psi_{1} \in L^{p}\left(\mathbb{R}^{n}\right)$ and $g * \Psi_{2} \in L^{q}\left(\mathbb{R}^{n}\right)$. Also, since $m \in \operatorname{BM}(p, q, r(x))$, we write $B_{m}\left(f * \Psi_{1}\right.$, $\left.g * \Psi_{2}\right)(y) \in L^{r(x)}\left(\mathbb{R}^{n}\right)$. Then, by the equality

$$
\left\|\tilde{B}_{m}\left(f * \Psi_{1}, g * \Psi_{2}\right)(y)\right\|_{r(x)}=\left\|B_{m}\left(f * \Psi_{1}, g * \Psi_{2}\right)(y)\right\|_{r(x)},
$$

the Hölder inequality and the inequality (3.5), we have

$$
\begin{aligned}
& \left|\int_{\mathbb{R}^{n}} \int_{\mathbb{R}^{n}} \hat{f}(\xi) \hat{g}(\eta) \hat{h}(\xi+\eta) \hat{\Psi}_{1}(\xi) m(\xi, \eta) \hat{\Psi}_{2}(\eta) d \xi d \eta\right| \\
& \quad \leq\|h\|_{r^{\prime}(x)}\left\|B_{m}\left(f * \Psi_{1}, g * \Psi_{2}\right)(y)\right\|_{r(x)} \\
& \quad \leq\|h\|_{r^{\prime}(x)}\left\|B_{m}\right\|\|f\|_{1}\left\|\Psi_{1}\right\|_{p}\|g\|_{1}\left\|\Psi_{2}\right\|_{q} .
\end{aligned}
$$

If we say $C=\left\|B_{m}\right\|\left\|\Psi_{1}\right\|_{p}\left\|\Psi_{2}\right\|_{q}$, we obtain

$$
\left|\int_{\mathbb{R}^{n}} \int_{\mathbb{R}^{n}} \hat{f}(\xi) \hat{g}(\eta) \hat{h}(\xi+\eta) \hat{\Psi}_{1}(\xi) m(\xi, \eta) \hat{\Psi}_{2}(\eta) d \xi d \eta\right| \leq C\|f\|_{1}\|g\|_{1}\|h\|_{r^{\prime}(x)} .
$$

Hence, $\hat{\Psi}_{1}(\xi) m(\xi, \eta) \hat{\Psi}_{2}(\eta) \in \mathrm{BM}(1,1, r(x))$.

(b) Take any $f, g, h \in C_{c}^{\infty}\left(\mathbb{R}^{n}\right)$. By (a), we know that

$$
\begin{aligned}
& \left|\int_{\mathbb{R}^{n}} \int_{\mathbb{R}^{n}} \hat{f}(\xi) \hat{g}(\eta) \hat{h}(\xi+\eta) \hat{\Psi}_{1}(\xi) m(\xi, \eta) \hat{\Psi}_{2}(\eta) d \xi d \eta\right| \\
& \quad \leq \int_{\mathbb{R}^{n}}|h(y)|\left|\tilde{B}_{m}\left(f * \Psi_{1}, g * \Psi_{2}\right)(y)\right| d x
\end{aligned}
$$

Similarly, if we say $C=\left\|B_{m}\right\|\left\|\Psi_{1}\right\|_{1}\left\|\Psi_{2}\right\|_{1}$, we obtain

$$
\left|\int_{\mathbb{R}^{n}} \int_{\mathbb{R}^{n}} \hat{f}(\xi) \hat{g}(\eta) \hat{h}(\xi+\eta) \hat{\Psi}_{1}(\xi) m(\xi, \eta) \hat{\Psi}_{2}(\eta) d \xi d \eta\right| \leq C\|f\|_{p}\|g\|_{q}\|h\|_{r^{\prime}(x)},
$$

which means $\hat{\Psi}_{1}(\xi) m(\xi, \eta) \hat{\Psi}_{2}(\eta) \in \operatorname{BM}(p, q, r(x))$.

In this theorem, (c) and (d) can be proved easily by using the technique of the proof in (a) and (b), respectively. 
Theorem 3.6 Let $m \in \mathrm{BM}\left(p_{1}(x), p_{2}(x), p_{3}(x)\right)$. If $Q_{1}, Q_{2} \subset \mathbb{R}^{n}$ are bounded sets, then

$$
h(\xi, \eta)=\frac{1}{\mu\left(Q_{1} \times Q_{2}\right)} \iint_{Q_{1} \times Q_{2}} m(\xi+u, \eta+v) d u d v \in \mathrm{BM}\left(p_{1}(x), p_{2}(x), p_{3}(x)\right) .
$$

Proof Let $f, g, h \in C_{c}^{\infty}\left(\mathbb{R}^{n}\right)$ be given. By using the Fubini theorem, we can easily prove the following equality:

$$
B_{h}(f, g)(x)=\frac{1}{\mu\left(Q_{1} \times Q_{2}\right)} \iint_{Q_{1} \times Q_{2}} B_{T_{(-u,-v) m}}(f, g)(x) d u d v .
$$

Also, by the Minkowski inequality and Theorem 3.3, we find $C_{0}>0$ such that

$$
\begin{aligned}
& \left\|B_{h}(f, g)\right\|_{p_{3}(x)} \\
& \quad \leq \frac{1}{\mu\left(Q_{1} \times Q_{2}\right)} C_{0} \iint_{Q_{1} \times Q_{2}}\left\|B_{T_{(-u,-v) m}}(f, g)\right\|_{p_{3}(x)} d u d v \\
& \leq \frac{1}{\mu\left(Q_{1} \times Q_{2}\right)} C_{0} \iint_{Q_{1} \times Q_{2}}\left\|T_{(-u,-v) m}\right\|_{\left(p_{1}(x), p_{2}(x), p_{3}(x)\right)}\|f\|_{p_{1}(x)}\|g\|_{p_{2}(x)} d u d v \\
& \quad=\frac{1}{\mu\left(Q_{1} \times Q_{2}\right)} C_{0}\|m\|_{\left(p_{1}(x), p_{2}(x), p_{3}(x)\right)}\|f\|_{p_{1}(x)}\|g\|_{p_{2}(x)} \mu\left(Q_{1} \times Q_{2}\right) \\
& \quad=C_{0}\|m\|_{\left(p_{1}(x), p_{2}(x), p_{3}(x)\right)}\|f\|_{p_{1}(x)}\|g\|_{p_{2}(x) .} .
\end{aligned}
$$

If we say $C=C_{0}\|m\|_{\left(p_{1}(x), p_{2}(x), p_{3}(x)\right)}$, we obtain

$$
\left\|B_{h}\right\|_{\left(p_{1}(x), p_{2}(x), p_{3}(x)\right)} \leq C\|f\|_{p_{1}(x)}\|g\|_{p_{2}(x)} .
$$

Hence $h(\xi, \eta) \in \mathrm{BM}\left(p_{1}(x), p_{2}(x), p_{3}(x)\right)$.

\section{Theorem 3.7}

(a) If $L^{s(x)}\left(\mathbb{R}^{n}\right) \subset L^{r(x)}\left(\mathbb{R}^{n}\right)$ and $m \in \mathrm{BM}(p(x), q(x), s(x))$, then $m \in \mathrm{BM}(p(x), q(x), r(x))$.

(b) If $L^{s(x)}\left(\mathbb{R}^{n}\right) \subset L^{r(x)}\left(\mathbb{R}^{n}\right), L^{p(x)}\left(\mathbb{R}^{n}\right) \subset L^{k(x)}\left(\mathbb{R}^{n}\right), L^{q(x)}\left(\mathbb{R}^{n}\right) \subset L^{t(x)}\left(\mathbb{R}^{n}\right)$ and $m \in \operatorname{BM}(k(x), t(x), s(x))$, then $m \in \operatorname{BM}(p(x), q(x), r(x))$.

Proof (a) Take any $f, g \in C_{c}^{\infty}\left(\mathbb{R}^{n}\right)$. Since $m \in \mathrm{BM}(p(x), q(x), s(x))$, there exists $C_{1}>0$ such that

$$
\left\|B_{m}(f, g)\right\|_{s(x)} \leq C_{1}\|f\|_{p(x)}\|g\|_{q(x)}
$$

Also, since $L^{s(x)}\left(\mathbb{R}^{n}\right) \subset L^{r(x)}\left(\mathbb{R}^{n}\right)$, there exists $C_{2}>0$ such that

$$
\left\|B_{m}(f, g)\right\|_{r(x)} \leq C_{2}\left\|B_{m}(f, g)\right\|_{s(x)} .
$$

If we set $C=C_{1} C_{2}$ and combine the inequalities (3.6) and (3.7), we have

$$
\left\|B_{m}(f, g)\right\|_{r(x)} \leq C\|f\|_{p(x)}\|g\|_{q(x)} .
$$

Therefore $m \in \mathrm{BM}(p(x), q(x), r(x))$. 
(b) Let us take any $f, g \in C_{c}^{\infty}\left(\mathbb{R}^{n}\right)$. Since $m \in \operatorname{BM}(k(x), t(x), s(x))$, there exists $C_{3}>0$ such that

$$
\left\|B_{m}(f, g)\right\|_{s(x)} \leq C_{3}\|f\|_{k(x)}\|g\|_{t(x)} .
$$

By using the inclusions $L^{s(x)}\left(\mathbb{R}^{n}\right) \subset L^{r(x)}\left(\mathbb{R}^{n}\right), L^{p(x)}\left(\mathbb{R}^{n}\right) \subset L^{k(x)}\left(\mathbb{R}^{n}\right)$ and $L^{q(x)}\left(\mathbb{R}^{n}\right) \subset L^{t(x)}\left(\mathbb{R}^{n}\right)$, we find $C_{4}, C_{5}, C_{6}>0$ such that

$$
\begin{aligned}
& \left\|B_{m}(f, g)\right\|_{r(x)} \leq C_{4}\left\|B_{m}(f, g)\right\|_{s(x)}, \\
& \|f\|_{k(x)} \leq C_{5}\|f\|_{p(x)}
\end{aligned}
$$

and

$$
\|g\|_{t(x)} \leq C_{6}\|g\|_{q(x)}
$$

If we set $C=C_{3} C_{4} C_{5} C_{6}$ and combine the inequalities (3.8), (3.9), (3.10) and (3.11), we have

$$
\left\|B_{m}(f, g)\right\|_{r(x)} \leq C\|f\|_{p(x)}\|g\|_{q(x)}
$$

Then $m \in \operatorname{BM}(p(x), q(x), r(x))$.

\section{Competing interests}

The authors declare that they have no competing interests.

\section{Authors' contributions}

The authors completed the paper together. They also read and approved the final manuscript.

\section{Acknowledgements}

Dedicated to Professor Hari M Srivastava.

This research was supported by the Ondokuz Mayıs University (PYO.FEN.1904.13.002).

Received: 23 November 2012 Accepted: 4 May 2013 Published: 23 May 2013

\section{References}

1. Feichtinger, H, Gürkanlı, AT: On a family of weighted convolution algebras. Int. J. Math. Math. Sci. 13(3), 517-526 (1990)

2. Fischer, RH, Gürkanlı, AT, Liu, TS: On a family of weighted spaces. Math. Slovaca 46(1), 71-82 (1996)

3. Katznelson, Y: An Introduction to Harmonic Analysis. Dover, New York (1968)

4. Reiter, H: Classical Harmonic Analysis and Locally Compact Groups. Oxford University Press, Oxford (1968)

5. Rudin, W: Fourier Analysis on Groups. Interscience, New York (1962)

6. Kovacik, O, Rakosnik, J: On spaces $L^{p(x)}$ and $W^{k, p(x)}$. Czechoslov. Math. J. 41(116), 592-618 (1991)

7. Aydın, I, Gürkanlı, AT: Weighted variable exponent amalgam spaces. Glas. Mat. 47(67), 165-174 (2012)

8. Aydın, I, Gürkanlı, AT: On some properties of the spaces $A_{\omega}^{p(x)}\left(\mathbb{R}^{n}\right)$. Proc. Jangjeon Math. Soc. 12(2), 141-155 (2009)

9. Gasquet, C, Witomski, P: Fourier Analysis and Applications. Springer, New York (1999)

10. Murthy, GNK, Unni, KR: Multipliers on weighted Spaces. In: Functional Analysis and Its Applications. Lecture Notes in Mathematics, vol. 399, pp. 272-291. Springer, Berlin (1973)

11. Blasco, O: Notes on the spaces of bilinear multipliers. Rev. Unión Mat. Argent. 50(2), 23-37 (2009)

12. Samko, SG: Convolution type operators in $L^{p(x)}$. Integral Transforms Spec. Funct. 7, 123-144 (1998)

doi:10.1186/1029-242X-2013-259

Cite this article as: Kulak and Gürkanlı: Bilinear multipliers of weighted Lebesgue spaces and variable exponent Lebesgue spaces. Journal of Inequalities and Applications 2013 2013:259. 